\title{
Optimal Uncertainty Quantification with Model Uncertainty and Legacy Data
}

\author{
P. H. T. Kamga, B. Li, M. McKerns, L. H. Nguyen, M. Ortiz, H. Owhadi, \\ T. J. Sullivan* \\ ${ }^{a}$ Division of Engineering and Applied Science, California Institute of Technology \\ Pasadena, CA91125, USA
}

\begin{abstract}
We present an Optimal Uncertainty Quantification (OUQ) protocol for systems that are characterized by an existing physics-based model and for which only legacy data is available, i. e., no additional experimental testing of the system is possible. Specifically, the OUQ strategy developed in this work consists of using the legacy data to establish, in a probabilistic sense, the level of error of the model, or modeling error, and to subsequently use the validated model as a basis for the determination of probabilities of outcomes. The quantification of modeling uncertainty specifically establishes, to a specified confidence, the probability that the actual response of the system lies within a certain distance of the model. Once the extent of model uncertainty has been established in this manner, the model can be conveniently used to stand in for the actual or empirical response of the system in order to compute probabilities of outcomes. To this end, we resort to the OUQ reduction theorem of Owhadi et al. [37] in order to reduce the computation of optimal upper and lower bounds on probabilities of outcomes to a finite-dimensional optimization problem. We illustrate the resulting UQ protocol by means of an application concerned with the response to hypervelocity impact of 6061-T6 Aluminum plates by Nylon 6/6 impactors at impact velocities in the range of $5-7 \mathrm{~km} / \mathrm{s}$. The ability of the legacy OUQ protocol to process diverse information on the system and its ability to supply rigorous bounds on system performance under realistic - and less than ideal - scenarios demonstrated by the hypervelocity impact application is remarkable.
\end{abstract}

${ }^{*}$ Corresponding author

E-mail address: ortiz@aero.caltech.edu (M. Ortiz).

Preprint submitted to Journal of the Mechanics and Physics of Solids

May 20, 2014

(C) 2014. This manuscript version is made available under the Elsevier user license http://www.elsevier.com/open-access/userlicense/1.0/ 
Keywords:

Hypervelocity impact, Uncertainty quantification, Optimal transportation, Meshfree interpolation, Particle erosion

\section{Introduction}

The principal objective of the present work is the formulation of a rigorous uncertainty quantification (UQ) protocol for systems characterized by a fixed data set, or legacy data, and by a physics-based model of unknown and uncertain fidelity, as well as with the formulation of efficient methods of solution and approximation thereof. By uncertainty quantification we specifically mean the determination of probabilities of outcomes in systems whose response is stochastic or uncertain, be it as a result of intrinsic randomness in the system, randomness in its inputs or operating conditions, epistemic uncertainty about system operation, or a combination thereof. A case in point concerns system certification, i. e., the assessment of the probability that a system will perform safely and within specifications. The system is certified if the probability of failure to perform safely is below a prespecified failure tolerance $\epsilon$. In addition, we wish to establish the confidence that we may place on a given certification assessment, i. e., we wish to ascertain the frequency with which the certification may be expected to be correct. Thus, we say that a system is certified with probability of failure $\epsilon$, or probability of success $1-\epsilon$, and confidence $1-\epsilon^{\prime}$ if the assessment that a system will perform successfully with probability $1-\epsilon$ is correct with probability $1-\epsilon^{\prime}$. Specifically, a way to test the preceding statement empirically is: i) Perform the certification assessment a large number of times; ii) For every certification assessment, test the system a large number of times and establish whether the certification assessment is correct, i. e., whether the system indeed fails with probability less than $\epsilon$; iii) Verify that the fraction of certifications that are correct is at least $1-\epsilon^{\prime}$. In practice, we often desire both $\epsilon$ and $\epsilon^{\prime}$ to be small numbers, i. e., we wish to certify with great confidence that the failure rate of the system is small. Indeed, a system can always be certified on the basis of overly-optimistic assumptions regarding its performance, but the confidence in such a certification should be correspondingly low. Conversely, insisting on a very high level of confidence in the certification assessment is bound to render certification unlikely. It is precisely the antagonistic demands of low failure tolerance and high certification confidence that make rigorous certification challenging and, in particular, place stringent requirements on experiment, modeling 
and uncertainty quantification.

In practice, a number of difficulties prevent the direct evaluation of the probability of failure of a system. For instance, the input space is often of very high dimension and some of the input parameters are unknown (socalled unknown unknowns). In addition, the probability measure of the known inputs - let alone the exact values of realizations of those inputs - is often not fully known. Many systems exhibit responses that are (or appear to be) stochastic in nature, and are controlled by physical processes whose functional form is only imperfectly characterized either by experiment or theory. Physical and computational models of the system may exist, but often these models are only partially verified and validated. In some cases, some of the underlying physics may be inadvertently omitted from the models or may not be covered by existing theory. This imperfect knowledge of the functional form of the response of the system is sometimes referred to as model uncertainty, and it constitutes a major source of uncertainty in practice. The situation is often compounded for systems whose performance cannot be fully tested, either because the operating conditions cannot be reproduced in the laboratory, or because the tests are costly, or subject to environmental or treaty restrictions, or some combination thereof. Often, legacy or archival data exists but is incomplete, inconsistent and noisy. Moreover, the mechanisms responsible for the failure of systems are often rare events and thus not directly accessible to simple Monte Carlo sampling; this inaccessibility makes it difficult to characterize their physical and probabilistic properties from experimental evidence. Finally, the failure of some systems is of great consequence, be it economic or in loss of life, and the tolerance for failure is correspondingly low. Here again, the tension between imperfect and incomplete information about the system and the desire to prevent rare but exceedingly costly failures during operation makes rigorous uncertainty quantification challenging in practice.

This work specifically considers systems that are characterized by an existing physics-based model and for which only legacy data is available, i. e., no additional experimental testing of the system is possible. The use of models for the design, assessment and certification of engineering systems is, evidently, standard. In addition, modeling and simulation is often less costly than experimental testing and lends itself to more extensive and systematic parametric studies. It is therefore desirable that a UQ protocol make maximum use of existing models while simultaneously ensuring rigorous and testable probabilities of outcomes and confidence levels in the assessment. Situations in which only legacy data is available also arise commonly in practice, e. g., in surveillance programs instituted to monitor the 
health of deployed systems, specially when a significantly abnormal condition is encountered in the field, and in the redesign of systems. In these cases, the questions of whether the existing data provide sufficient coverage of the system under abnormal conditions, or of the redesigned system, and the extent to which designs can be 'extrapolated' away from the legacy data base, are of central concern.

The UQ strategy adopted in this work consists of using the legacy data to establish, in a probabilistic sense, the level of error of the model, or modeling error, and to subsequently use the validated model as a basis for the determination of probabilities of outcomes. The quantification of modeling uncertainty specifically establishes, to a specified confidence, the probability that the actual response of the system, which may depend on hidden or unaccounted-for variables, lie within a certain distance of the model. Once the extent of model uncertainty has been established in this manner, the model can be conveniently used to stand in for the actual or empirical response of the system in order to compute probabilities of outcomes. Evidently, in this approach model uncertainty compounds uncertainties due to the intrinsic randomness of the system or to the randomness of the inputs. Therefore, in practice the success and the proposed UQ protocol depends critically on the fidelity of the model and the quality and extent of the data. Once the model has been validated, and the model uncertainty has been quantified, we compute bounds on probabilities of outcomes by recourse to Optimal Uncertainty Quantification (OUQ) [37]. In particular, we resort to the reduction theorem of Owhadi et al. [37] in order to reduce the computation of optimal upper and lower bounds on probabilities of outcomes to a finite-dimensional optimization problem.

We illustrate the resulting UQ protocol by means of an application concerned with the response to hypervelocity impact of 6061-T6 Aluminum plates by Nylon 6/6 impactors at impact velocities in the range of $5-7 \mathrm{~km} / \mathrm{s}$. Hypervelocity impact sets in motion complex physics that challenge modeling and simulation, both as regards material modeling and solvers. The system under consideration is characterized by three input parameters, namely, impact velocity, plate thickness, and obliquity, and the outcome of interest (i. e. quantity of interest) is the perforation area. The resulting UQ problem concerns, therefore, the determination of bounds on perforation area probabilities when the system is allowed to operate over a certain range of the input parameters. Experimental data in support of the UQ analysis is obtained at Caltech's Small Particle Hypervelocity Impact Range (SPHIR) $[1,35,34]$. Specifically, a legacy data set is generated from 65 hypervelocity shots providing coverage over the range of inputs. In addition, we 
employ physics-based, phase-aware models of 6061-T6 Al and Nylon 6/6 in conjunction with the Optimal Transportation Meshfree (OTM) [25, 26] Lagrangian solver, which includes fragmentation and contact capability, in order to model the hypervelocity tests. The level of model uncertainty is then quantified in a probabilistic sense by comparing the model against the legacy data. Finally, optimal upper bounds on perforation-area probabilities, with or without additional a priori information in the form of input and output moment constraints, are obtained using the mystic framework $[32,33]$ in order to carry out the requisite optimization calculations. Overall, the ability of OUQ to process diverse information on the system and its ability to supply rigorous bounds on system performance under realistic - and less than ideal-scenarios is remarkable.

\section{Model-driven Optimal Uncertainty Quantification with legacy data}

Suppose that we wish to certify of a system, i. e., we wish to ascertain the probability that the system operate safely and within design specifications. A number of uncertainties compound the determination of such probability. Thus, the frequency with which different operating conditions arise in the field is likely to be uncertain, and the response of the system is likely to be stochastic. In addition, the system is likely to be characterized by a limited legacy data set exhibiting a certain amount of scatter. Physics-based computational models may exist but such models often exhibit a certain level of modeling error. The challenge, therefore, is to establish rigorous bounds on probabilities of outcomes of the system under such less-than-ideal, albeit realistic, conditions. To this end, we rely of the theory of Optimal Uncertainty Quantification of Owhadi et al. [37], which we specialize, and also extend, to encompass the scenario under consideration.

\subsection{General framework}

We begin by summarizing the relevant aspects of the theory of Optimal Uncertainty Quantification (OUQ) [37] for subsequent reference. We also proceed to specialize the general OUQ framework to the setting of interest in the present work, namely, systems that are presumed to be wellcharacterized by an available model and for which only legacy data is available, i. e., no additional experimental testing of the system is possible. We consider throughout a system with random inputs in a space of inputs $\mathcal{X}$, whose behavior is characterized by a response function $F: \mathcal{X} \rightarrow \mathcal{Y}$ that takes values in a space of outputs $\mathcal{Y}$. In order to account for uncertainties in the 
system inputs $X$ in $\mathcal{X}$, commonly referred to as aleatoric uncertainties, we treat them as random variables with probability measure $\mathbb{P}$. The points of $\mathcal{Y}$ represent a collection of performance measures, and the safe behavior of the system requires that the induced random variable $F(X)$ take values within a safe set $A \subseteq \mathcal{Y}$. Correspondingly, the system fails if the value of $F(X)$ lies in the complement $A^{c}=\mathcal{X} \backslash A \equiv U$, or unsafe set. Therefore, the probability of failure of the system is $\mathbb{P}(\{x \in \mathcal{X} \mid F(x) \notin A\})$, or $\mathbb{P}[F(X) \notin A]$ for short. We say that the system is certified with probability at least $1-\epsilon$ if its probability of failure satisfies $\mathbb{P}[F(X) \notin A] \leq \epsilon$, where $\epsilon$ denotes the failure tolerance.

Following Owhadi et al., suppose that the available information about a system is limited and does not uniquely (i. e., perfectly) specify the real response function $F$ and which probability measure $\mathbb{P}$. Instead, the available information suffices only to restrict the possible response functions $F$ and the possible probability measures $\mathbb{P}$ of the inputs to some admissible set or information set $\mathcal{A}$. Thus, a pair $(f, \mu) \in \mathcal{A}$ represents a possible response function $f$ and a possible input probability measure $\mu$ consistent with all the information available on the system. Under these conditions, we wish to determine the best, or tightest, upper and lower bounds on the probability of failure of the system, namely

$\mathcal{L}(\mathcal{A}) \equiv \inf _{(f, \mu) \in \mathcal{A}} \mathbb{P}_{\mu}[f(X) \notin A] \leq \mathbb{P}[F(X) \notin A] \leq \sup _{(f, \mu) \in \mathcal{A}} \mathbb{P}_{\mu}[f(X) \notin A] \equiv \mathcal{U}(\mathcal{A})$

The bounds (1) are indeed optimal in the sense that if $p_{L}$ and $p_{U}$ are respectively any valid lower and upper bounds on the probability of failure, it follows that

$$
p_{L} \leq \mathcal{L}(\mathcal{A}) \leq \mathbb{P}[F(X) \notin A] \leq \mathcal{U}(\mathcal{A}) \leq p_{U} .
$$

In particular, the condition $\mathcal{U}(\mathcal{A}) \leq \epsilon$ provides a healthily conservative (with respect to $\mathcal{A}$ ) certification criterion in lieu of the unknown exact probability of failure $\mathbb{P}[F(X) \in A]$. We also note that the optimal bounds $\mathcal{L}(\mathcal{A})$ and $\mathcal{U}(\mathcal{A})$ have the following monotonicity property: if additional information is supplied on the system so that the new admissible set is $\mathcal{B} \subseteq \mathcal{A}$, then

$$
\mathcal{L}(\mathcal{A}) \leq \mathcal{L}(\mathcal{B}) \leq \mathbb{P}[F(X) \notin A] \leq \mathcal{U}(\mathcal{B}) \leq \mathcal{U}(\mathcal{A})
$$

i. e., the availability of additional information has the effect of improving - or tightening — the bounds. Naturally, the inequality (3) only holds because the information describing $\mathcal{B}$ extends and does not contradict the information describing $\mathcal{A}$; otherwise, it may be that no inequality of the 
min and max of

$\mathbb{P}_{\mu}[g(X) \leq t]$

over $(g, \mu) \in \mathcal{A}$

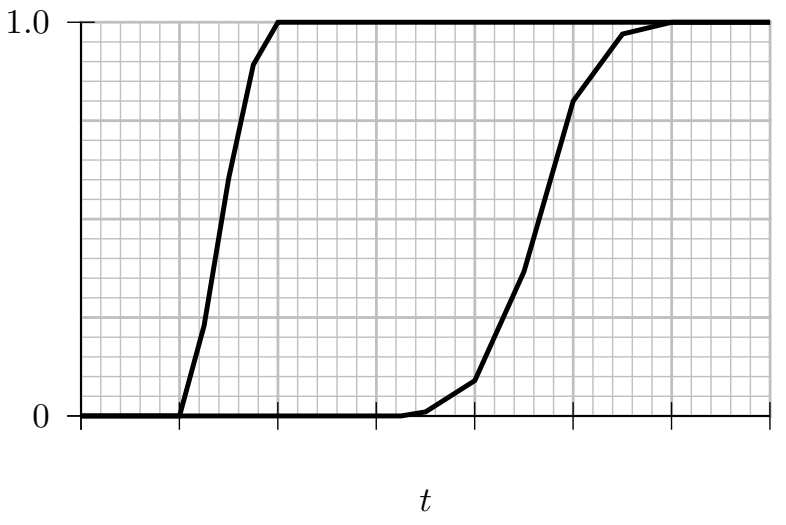

Figure 1: A schematic illustration of OUQ lower and upper envelopes for the CDF of the system response.

form (3) holds. In particular, in the case of a contradiction we obtain the pathological special case $\mathcal{L}(\mathcal{A} \cap \mathcal{B})=1$ and $\mathcal{U}(\mathcal{A} \cap \mathcal{B})=0$ (sic).

It is worth noting that, in practice, one is often interested not just in $\mathbb{P}[F(X) \notin A]$ for a single safe set $A$, but a family of such sets. For example, if $F$ takes real values, one may be interested in $\mathbb{P}[F(X) \leq t]$ as a function of $t$, i. e., the cumulative distribution function (CDF) of the output random variable $F(X)$. In this setting, plots of $\mathcal{L}(\mathcal{A})$ and $\mathcal{U}(\mathcal{A})$ as a function of $t$ provide lower and upper envelopes for the true but unknown CDF, as shown in schematic form in Figure 1; any increasing function within that envelope could be the CDF of $F(X)$, but the information encoded in $\mathcal{A}$ does not allow us to know which one, and in high-consequence decision-making settings it would be unwise to arbitrarily select one.

The preceding OUQ framework described so far presumes that a welldefined admissible set $\mathcal{A}$ is available and that it is possible to determine unambiguously whether a given pair $(f, \mu)$ belongs or not to the set. However, in many practical situations the admissible set $\mathcal{A}$ is only known in a probabilistic or statistical sense: all that can be stated is that the actual response function of the system $F$ and the input probability measure $\mathbb{P}$ are in $\mathcal{A}$ with probability $1-\epsilon^{\prime}$. A case in point concerns systems characterized by legacy data, which of necessity provide only partial - and often noisy - coverage of the response of the system. In such cases, the optimal lower and upper bounds (1) bound the probability of failure $\mathbb{P}[F(X) \notin A]$ with probability $1-\epsilon^{\prime}$. Correspondingly, if, as before, $\epsilon$ is a failure tolerance and if $\mathcal{U}(\mathcal{A}) \leq \epsilon$, we may then conclude that the system is certified with $1-\epsilon^{\prime}$ 
confidence, i. e., that the probability of failure of the system is less than $\epsilon$ with probability $1-\epsilon^{\prime}$.

Evidently, $1-\epsilon^{\prime}$ measures the confidence that can be placed on the certification process. Thus, in the absence of data, whereas a system may be formally certified on the basis of assumed or hypothesized input probabilities and models, no confidence can be placed on the certification. Conversely, the higher the certainty $1-\epsilon^{\prime}$ on models and input probabilities, e. g., resulting from increased data coverage, the higher the confidence that can be placed on the corresponding certification. Thus, the probabilistic OUQ framework effectively quantifies the adverse effect on certification of model uncertainty and extrapolation from legacy data.

\subsection{The reduction theorem}

A priori, the computation of $\mathcal{L}(\mathcal{A})$ and $\mathcal{U}(\mathcal{A})$ entails the solution of two global optimization problems over infinite dimensional spaces of functions and measures. However, it has been shown by Owhadi et al. [37] that these optimization problems can be reduced to equivalent finite-dimensional problems, and that the resulting finite-dimensional problems are computable. Specifically, the reduction theorem of Owhadi et al. states that, under rather general conditions, $\mathcal{L}(\mathcal{A})$ and $\mathcal{U}(\mathcal{A})$ can be obtained by restricting the minimization and maximization of the probability of failure to a reduced admissible set $\mathcal{A}_{\Delta}$ consisting of probability measures of the form

$$
\mu \in\left\{\begin{array}{l|l}
\sum_{j=0}^{k} \alpha_{j} \delta_{x_{j}} & \begin{array}{c}
\text { for some } x_{0}, \ldots, x_{k} \in \mathcal{X}, \\
\text { and some } \alpha_{0}, \ldots, \alpha_{k} \geq 0, \\
\text { such that } \sum_{j=0}^{k} \alpha_{j}=1,
\end{array}
\end{array}\right\},
$$

i. e., finite convex combinations of at most $k+1$ Dirac masses. The number of Dirac masses that it is sufficient to consider in the optimization is also established by the reduction theorem: if the admissible set $\mathcal{A}$ is defined by means of $k$ constraints on expected inputs and/or outputs, then it is sufficient to consider $k+1$ Dirac masses in the optimization. A precise statement of the reduction theorem is included in the Appendix for completeness. For probability measures of the form (4), we have

$$
\mathbb{P}_{\mu}[f(X) \notin A]=\sum_{j=0}^{k} \alpha_{j} c_{j}, \quad \text { where } c_{j}= \begin{cases}1, & \text { if } f\left(x_{j}\right) \notin A, \\ 0, & \text { otherwise. }\end{cases}
$$

The optimal bounds $\mathcal{L}(\mathcal{A})$ and $\mathcal{U}(\mathcal{A})$ now follow by minimizing and maximizing the trial probability of failure (5) with respect to values $\left(\alpha_{j}, x_{j}\right)_{j=0}^{k}$ 
consistent with the constraints, and with respect to admissible models $f$. We note that, for fixed $f$ and $\left(x_{j}\right)_{j=0}^{k}$, the extremization of probability of failure with respect to $\left(\alpha_{j}\right)_{j=0}^{k}$ reduces to a standard linear programming problem.

The reduction theorem of Owhadi et al. [37] applies for general constraints on the space of possible models and for independent inputs $X_{1}, \ldots, X_{m}$ taking values in a product space $\mathcal{X}=\mathcal{X}_{1} \times \cdots \times \mathcal{X}_{m}$ subject to constraints of the form

$$
\begin{array}{r}
\mathbb{E}_{\mu}\left[g_{0 j}\right] \leq 0, \quad \text { for } j=1, \ldots, n_{0}, \\
\mathbb{E}_{\mu_{1}}\left[g_{1 j}\right] \leq 0, \quad \text { for } j=1, \ldots, n_{1}, \\
\vdots \\
\mathbb{E}_{\mu_{m}}\left[g_{m j}\right] \leq 0, \quad \text { for } j=1, \ldots, n_{m},
\end{array}
$$

where $g_{0 j}: \mathcal{X} \rightarrow \mathbb{R}\left(\right.$ for $\left.j=1, \ldots, n_{0}\right)$ and $g_{i j}: \mathcal{X}_{i} \rightarrow \mathbb{R}$ (for $i=1, \ldots, m$ and $\left.j=1, \ldots, n_{i}\right)$ are measurable functions that may depend in an arbitrary way on $f$. Thus, a typical admissible set is of the form

$$
\mathcal{A}=\left\{(\mu, f) \mid \begin{array}{c}
f \in \mathcal{F} \\
\mu=\mu_{1} \otimes \cdots \otimes \mu_{m}, \\
\mathbb{E}_{\mu}\left[g_{0 j}\right] \leq 0 \text { for } j=1, \ldots, n_{0}, \\
\mathbb{E}_{\mu_{1}}\left[g_{1 j}\right] \leq 0 \text { for } j=1, \ldots, n_{1}, \\
\vdots \\
\mathbb{E}_{\mu_{m}}\left[g_{m j}\right] \leq 0 \text { for } j=1, \ldots, n_{m}
\end{array}\right\}
$$

where $\mathcal{F}$ is some set of measurable functions defining a range of possible response functions. (Recall here that the statement that the components $X_{1}, \ldots, X_{m}$ of a random variable $X=\left(X_{1}, \ldots, X_{m}\right)$ are independent random variables is equivalent to the statement that the joint distribution $\mu$ of $X$ is the product $\mu=\mu_{1} \otimes \cdots \otimes \mu_{m}$ of the marginal distributions $\mu_{i}$ of each $X_{i}$.)

The type of constraints on the input probability measure contemplated under the reduction theorem of Owhadi et al. [37] include, but are not limited to, simple moment constraints on the inputs and on the outputs, in which case the constraint functions $g_{0 j}$ are polynomials over $\mathcal{X}$ or over $\mathcal{Y}$, respectively. Linear moment constraints on the factor spaces $\mathcal{X}_{i}$ permit consideration of information sets with independent random variables $X_{1}, \ldots, X_{m}$ and weak constraints on their probability distribution. An elementary example of such an admissible set is furnished by Chebyshev's 
inequality, in which a priori bounds are given on the mean and variances of a single random variable $X$ and are used to bound its tail probabilities. Other examples of information sets of practical relevance arise, e. g., when a priori bounds are known on the mean performance $\mathbb{E}_{\mu}[f]$ of the system or on the oscillation of the system response $[37,23,1]$. The optimal bounds delivered by OUQ in these cases improve on classical inequalities such as Hoeffding's [21] and McDiarmid's [31]. A different type of constraints on the information set arises when certain features of the response function $f$ can be elucidated a priori, e. g., Lipschitz or Hölder constants, and when legacy or archival experimental data is available [52]. Issues pertaining to the computability of the optimal bounds, and efficient algorithms for their computation thereof, are discussed in $[37,23,1]$.

\subsection{Definition of the admissible set}

We now turn to the crucial question of characterizing the admissible set $\mathcal{A}$ of possible response functions and input probability measures. We specifically direct our attention to the scenario, commonly encountered in practice, in which the response of the system is characterized by a fixed set of experimental data, or legacy data set. We assume, in addition, the availability of a validated model that approximates the response of the system to a high level of fidelity. We proceed to show that it is possible to use the legacy data in order to establish rigorous confidence intervals around the

model, i. e., to bound from below the probability that the real response of the system differ from the model by less than a certain offset, or modeling error. This strategy in effect characterizes the admissible set $\mathcal{A}$ in probabilistic terms, and provides a valuable measure of model uncertainty. Once this level of model uncertainty is quantified, the OUQ machinery described in the foregoing can be applied to certify systems with quantified confidence.

\subsubsection{Metrization of the space of models}

In order to establish confidence intervals around a given model, we need to metrize the space of response functions, i. e., we need to supply a means of measuring distances between response functions. An obvious first candidate for such a distance is the uniform distance (or supremum norm): we assume that we have a model $F_{M}: \mathcal{X} \rightarrow \mathcal{Y}$ for the true response $F$ and that we have to hand an upper bound on the uniform distance

$$
d_{\mathrm{U}}\left(F_{M}, F\right) \equiv\left\|F_{M}-F\right\|_{\infty} \equiv \operatorname{ess} \sup _{x \in \mathcal{X}} d_{\mathcal{Y}}\left(F_{M}(x), F(x)\right),
$$

where $d_{\mathcal{Y}}$ is some distance function (metric) on $\mathcal{Y}$. That is, we assume that the partially known reality $F$ lies close to the model function $F_{M}$. However, 


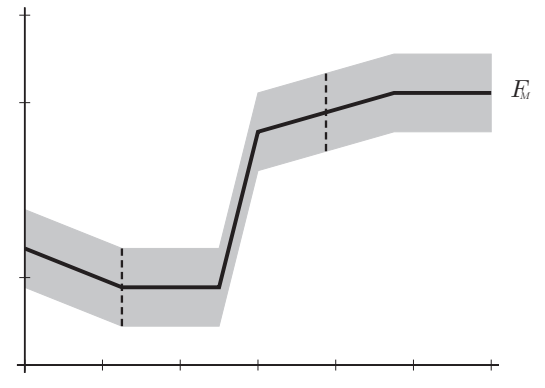

(a)

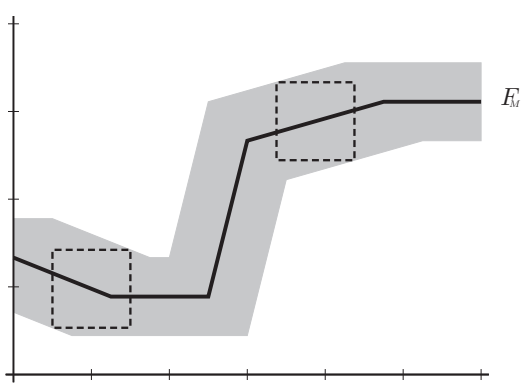

(b)

Figure 2: Schematic illustration of (a) uniform and (b) Hausdorff neighborhoods of function. Note the relative sizes of the two neighborhoods near the function's cliff.

despite its simplicity, the uniform distance is not a practical choice for the kinds of systems often encountered in practice that have sharp cliffs or even discontinuities. As shown in Figure 2, near such cliffs, the actual size of the uniform neighborhood of $F_{M}$ is tiny, because although its "vertical" width in $\mathcal{Y}$ remains constant, its "horizontal" width in $\mathcal{X}$ is very small. To assume that $d_{\mathrm{U}}\left(F, F_{M}\right)$ is small is to assume that $F_{M}$ has approximately the same features as $F$ but in exactly the same places - often an unreasonably strong assumption. Therefore, we seek a notion of distance between $F_{M}$ and $F$ that encapsulates the notion that $F_{M}$ has approximately the same features as $F$ and in approximately the same places.

To this end, suppose that the Cartesian product space $\mathcal{X} \times \mathcal{Y}$ is jointly metrized by a distance $d$. Let $f: \mathcal{X} \rightarrow \mathcal{Y}$ and $g: \mathcal{X} \rightarrow \mathcal{Y}$ be two functions, let $\Gamma_{f}=\{(x, f(x)) \in \mathcal{X} \times \mathcal{Y} \mid x \in \mathcal{X}\}$ denote the graph of $f$, and $\Gamma_{g}$ similarly. We recall that the Hausdorff semi-distance from one set $A$ to another set $B$ in a metric space $(M, d)$ is

$$
d_{\mathrm{H}}(A, B)=\sup _{x \in A} \inf _{y \in B} d(x, y) .
$$

This semi-distance is not symmetric (in general, $d_{\mathrm{H}}(A, B) \neq d_{\mathrm{H}}(B, A)$ ), and so the Hausdorff distance between $A$ and $B$ is defined to be the maximum of $d_{\mathrm{H}}(A, B)$ and $d_{\mathrm{H}}(B, A)$. Correspondingly, we define the Hausdorff semidistance from the function $f$ to the function $g$ as

$$
d_{\mathrm{H}}(f, g) \equiv d_{\mathrm{H}}\left(\Gamma_{f}, \Gamma_{g}\right)=\sup _{x \in \mathcal{X}} \inf _{x^{\prime} \in \mathcal{X}} d\left((x, f(x)),\left(x^{\prime}, g\left(x^{\prime}\right)\right)\right.
$$


As a particular case, if $\left(\mathcal{Y}, d_{\mathcal{Y}}\right)$ is a metric space and we set

$$
d\left((x, y),\left(x^{\prime}, y^{\prime}\right)\right)= \begin{cases}d \mathcal{Y}\left(y, y^{\prime}\right), & \text { if } x^{\prime}=x \\ +\infty, & \text { otherwise }\end{cases}
$$

then the Hausdorff semi-distance reduces to the uniform distance defined in (8). Figure 2 shows how the Hausdorff neighborhood of a function is large than its uniform neighborhood, even near cliffs; hence, while an assumption of the type $d_{\mathrm{H}}\left(F, F_{M}\right) \leq \delta$ is looser than one of the type $d_{\mathrm{U}}\left(F, F_{M}\right) \leq \delta$, and hence leads to a wider interval bound $[\mathcal{L}(\mathcal{A}), \mathcal{U}(\mathcal{A})]$ on the probability of failure, the assumption that $d_{\mathrm{H}}\left(F, F_{M}\right) \leq \delta$ is much more likely to be valid.

We note that the choice of distance $d$ is to some extent arbitrary, in the same way that choices of physical units are arbitrary. A situation commonly encountered in practice concerns inputs and outputs that are physically disparate and, in particular, are measured in different units. Thus, whereas each individual input $X_{i}$ and output $Y_{j}$ can be measured unambiguously, there is no natural distance defined on $\mathcal{X}, \mathcal{Y}$ or $\mathcal{X} \times \mathcal{Y}$; obvious choices include the sum and the maximum of the componentwise distances. A open question concerns the optimal choice of joint metric on $\mathcal{X} \times \mathcal{Y}$ that results in the highest possible certification confidence for a given model and legacy data set.

\subsubsection{Confidence intervals around the model}

Let $F_{M}: \mathcal{X} \rightarrow \mathcal{Y}$ be a model. One way to use the model $F_{M}$ to characterize, albeit probabilistically, the admissible set $\mathcal{F}$ of possible response functions, cf. eq. (7), is to obtain a lower bound on the probability that the model $F_{M}$ differ from the actual response $F$ of the system by less than a given modeling error. Specifically, we wish to derive, with a prespecified level of confidence $1-\epsilon^{\prime}$, a lower bound on the probability

$$
p_{\delta} \equiv \mathbb{P}\left[d\left((X, Y), \Gamma_{F_{M}}\right) \leq \delta\right]
$$

where $\delta>0$ and $(X, Y)$ is the random vector $(X, F(X))$ taking values in $\mathcal{X} \times \mathcal{Y}$ and corresponding to the random realization of the input variable $X$ and the response $F(X)$ of the system of interest. More precisely, we wish to obtain intervals of confidence around the model $F_{M}$ for use in OUQ analysis. It should be noted that the method presented here does not assume that all the input variables are known, i. e., $F(X)$ given the value of $X$ may be stochastic owing to, e. g., random unknown unknowns.

Suppose that we observe or are given $\left\{\left(X_{i}, Y_{i}\right) \in \mathcal{X} \times \mathcal{Y}, i=1, \ldots, n\right\}$, i. e., $n$ independent and identically distributed (i.i.d.) experimental realizations of samples from $(X, Y)$. Thus, $\left(X_{i}, Y_{i}\right)$ represents the $i$ th data point 
in an experimental data repository. For the purpose of obtaining a lower bound on (12) from the data set we introduce the random variables

$$
Z= \begin{cases}1, & \text { if } d\left((X, Y), \Gamma_{F_{M}}\right) \leq \delta \\ 0, & \text { otherwise }\end{cases}
$$

and

$$
Z_{i}= \begin{cases}1, & \text { if } d\left(\left(X_{i}, Y_{i}\right), \Gamma_{F_{M}}\right) \leq \delta \\ 0, & \text { otherwise }\end{cases}
$$

We observe that $Z$ and $Z_{1}, \ldots, Z_{n}$ are $n+1$ i.i.d. Bernoulli random variables equal to one with probability $p_{\delta}$ and zero with probability $1-p_{\delta}$. A natural estimation of the $p_{\delta}$ is given by the empirical mean

$$
\bar{Z} \equiv \frac{1}{n} \sum_{i=1}^{n} Z_{i} .
$$

To obtain a lower bound on (12) it is therefore sufficient to bound the probability of deviation of the empirical mean $\bar{Z}$ from its average $\mathbb{E}[Z]$, i. e., a lower bound on $\mathbb{P}\left[p_{\delta} \geq \bar{Z}-r\right]$. Since $n \bar{Z}$ is a binomial distribution with parameters $p_{\delta}$ and $n$, we have

$$
\mathbb{P}\left[p_{\delta} \geq \bar{Z}-r\right]=\mathbb{P}\left[n\left(p_{\delta}+r\right) \geq B\left(n, p_{\delta}\right)\right],
$$

where, by a standard abuse of notation, we write $B(n, p)$ for a random variable having binomial distribution with parameters $(p, n)$. Given the lack of information on $p_{\delta}$ the optimal lower bound on $\mathbb{P}\left[n\left(p_{\delta}+r\right) \geq B\left(n, p_{\delta}\right)\right]$, and therefore $\mathbb{P}\left[p_{\delta} \geq \bar{Z}-r\right]$, is

$$
\eta_{n, r} \equiv \inf _{q \in[0,1]} \mathbb{P}[n(q+r) \geq B(n, q)] .
$$

The infimum is attained for $q=1 / 2$ and takes the value

$$
\eta_{n, r}=\mathbb{P}[n(1 / 2+r) \geq B(n, 1 / 2)] .
$$

We thus conclude that

$$
\mathbb{P}\left[d\left((X, Y), \Gamma_{F_{M}}\right) \leq \delta\right] \geq \bar{Z}-r
$$

with probability at least $\eta_{n, r}$. Note that since $\bar{Z}$ is a function of the experimental data it is stochastic in nature and the inequality (19) may or may not hold. However, the preceding analysis shows that $\eta_{n, r}$ is an optimal lower bound on the probability that the inequality (19) hold. We may thus 


\begin{tabular}{|c||c|c|c|c|c|c|}
\hline$\eta_{n, r}$ & $r=0.00$ & $r=0.02$ & $r=0.04$ & $r=0.06$ & $r=0.08$ & $r=0.10$ \\
\hline$n=100$ & 0.5 & 0.617823 & 0.757941 & 0.864373 & 0.933395 & 0.971556 \\
\hline$n=200$ & 0.5 & 0.689636 & 0.85559 & 0.948180 & 0.985937 & 0.997157 \\
\hline$n=300$ & 0.5 & 0.737279 & 0.907942 & 0.978436 & 0.996720 & 0.999683 \\
\hline$n=400$ & 0.5 & 0.773352 & 0.939484 & 0.990669 & 0.999200 & 0.999963 \\
\hline$n=500$ & 0.5 & 0.802245 & 0.959484 & 0.995869 & 0.999800 & 0.999996 \\
\hline$n=600$ & 0.5 & 0.826123 & 0.972538 & 0.998143 & 0.999949 & 0.999999 \\
\hline$n=700$ & 0.5 & 0.846256 & 0.981220 & 0.999156 & 0.999987 & 1. \\
\hline$n=800$ & 0.5 & 0.863469 & 0.987070 & 0.999614 & 0.999997 & 1. \\
\hline$n=900$ & 0.5 & 0.878335 & 0.991050 & 0.999822 & 0.999999 & 1. \\
\hline$n=1000$ & 0.5 & 0.891276 & 0.993778 & 0.999917 & 1. & 1. \\
\hline
\end{tabular}

Table 1: Optimal lower bound $\eta_{n, r}$ on the probability that the probability $\mathbb{P}\left[\Gamma_{F} \in \mathcal{B}\right]$ that the graph $\Gamma_{F}$ of the response function $F$ be in $\mathcal{B} \subset \mathcal{X} \times \mathcal{Y}$ be at least $\bar{Z}-r$, where $n=\# \mathcal{D}$ is the size of the data set $\mathcal{D}$ and $\bar{Z}=\#(\mathcal{B} \cap \mathcal{D}) / n$.

take $\eta_{n, r}$ to define the level of confidence that we may place on inequality (19). Note, however, that the definition of an acceptable value for $\eta_{n, r}$ (just like the definition of $\epsilon$, the maximum acceptable probability of failure) is a 'policy decision' to be made by the decision-making users of the UQ analysis, not a mathematical judgement to be made within the UQ analysis itself.

A sampling of representative values of $\eta_{n, r}$ are shown in Table 1 by way of illustration. In general, for purposes of certification we wish the probability $p_{\delta}$ that the actual response of the system be within a $\delta$-neighborhood $\left\{d\left((X, Y), \Gamma_{F_{M}}\right) \leq \delta\right\}$ of the model, or its lower bound $\bar{Z}-r$ thereof, to be high. We may take $\delta$ as a measure of the modeling error that is allowed for in the OUQ analysis. Evidently, $\bar{Z}-r$ is high is achieved if $\bar{Z}$ is close to 1 , i. e., if the model fits the data closely and if $\delta$ is chosen large enough that the corresponding $\delta$-neighborhood encompasses the majority of the data, in which case $\bar{Z}$ is close to 1 . However, by increasing $\delta$ we may correspondingly allow for large deviations of the response of the system from the model in the OUQ analysis, with the result of increasing the probability of failure and reducing the certifiability of the system. An alternative means of increasing $\bar{Z}-r$ is to reduce the slack $r$. However, this reduction in turn has the effect of reducing the level of confidence $\eta_{n, r}$ that we can have on $\bar{Z}-r$ being a lower bound on $p_{\delta}$ and, by extension, on the certification outcome. Contrariwise, such level of confidence is increased by increasing the size $n$ of the experimental sample. These opposing trends set forth trade-offs between modeling error, the size of the data set, and confidence in the certification 
process that need to be carefully evaluated in practice.

\subsubsection{Confidence intervals for means and variances}

The admissible set $\mathcal{A}$ can also be characterized by obtaining confidence bounds on the means or variances of input variables and/or the response function $F$. Such bounds can be obtained through Hoeffding's inequality. For the sake of completeness, we recall this inequality here. Let $X$ and $X_{1}, \ldots, X_{n}$ be $n$ i.i.d. real random variables with values in $[a, b]$. We denote the sample mean as $\bar{X} \equiv \frac{1}{n} \sum_{i=1}^{n} X_{i}$. For a positive real $t$, Hoeffding's inequality states that

$$
\mathbb{P}\{|\bar{X}-\mathbb{E}[X]| \geq t\} \leq 2 \exp \left(\frac{-2 n t^{2}}{(b-a)^{2}}\right)
$$

Let $\gamma \in[0,1]$ and define

$$
t_{\gamma} \equiv(b-a) \sqrt{\frac{\log \left(\frac{2}{1-\gamma}\right)}{2 n}}
$$

Inequality (20) can then be used to deduce that the probability that the expected value $\mathbb{E}[X]$ lies within the confidence interval $\mathbb{E}[X] \in\left[\bar{X}-t_{\gamma}, \bar{X}+\right.$

$\left.t_{\gamma}\right]$, is at least $\gamma$. Recall that the variance $X$ is $\mathbb{E}\left[X^{2}\right]-(\mathbb{E}[X])^{2}$. Since Hoeffding's inequality can also be used to obtain confidence intervals on mean of $X^{2}$, Hoeffding's inequality can be used as described above to obtain confidence intervals on the variance of $X$.

\section{Application to hypervelocity impact}

We proceed to demonstrate the legacy OUQ protocol described in foregoing through an application to hypervelocity impact. In this application, the system is characterized by three input parameters, namely, target plate thickness $h$, impact velocity $v$ and obliquity $\alpha$, with assumed ranges $h \in \mathcal{X}_{h} \equiv\{0.5 \mathrm{~mm}, 1.5 \mathrm{~mm}, 3.0 \mathrm{~mm}\}, v \in \mathcal{X}_{v} \equiv\left[4.5 \mathrm{~km} . \mathrm{s}^{-1}, 7.0 \mathrm{~km} . \mathrm{s}^{-1}\right]$ and $\alpha \in \mathcal{X}_{\alpha} \equiv\left[0^{\circ}, 60^{\circ}\right]$. The corresponding input space is, therefore, $\mathcal{X} \equiv \mathcal{X}_{h} \times \mathcal{X}_{v} \times \mathcal{X}_{\alpha}$. For definiteness, we choose the perforation area as the main performance measure of the system, which is expected to lie in the range $\mathcal{Y} \equiv\left[0,39.73 \mathrm{~mm}^{2}\right]$. The response of the system in this application is, therefore, the function that maps impact velocity, plate thickness and obliquity to perforation area. This response function exhibits challenging features, such as threshold value of the impact velocity, or ballistic limit, below which the perforation area is zero, a sharp increase in perforation 
area above the ballistic limit, or cliff behavior, and eventually a plateau where the dependence of perforation area on impact velocity exhibits comparatively smaller variation.

\subsection{Hypervelocity impact experiments}

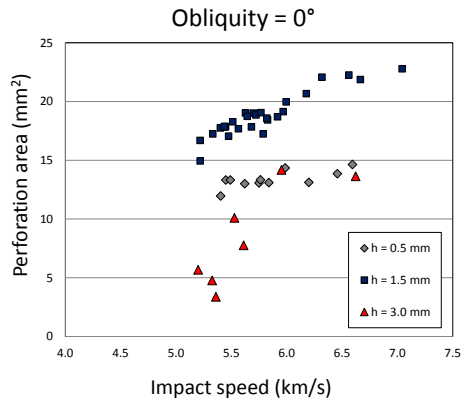

(a)

\begin{tabular}{cccc}
\hline thickness [mm] & obliquity $[\mathrm{deg}]$ & velocity $[\mathrm{km} / \mathrm{s}]$ & Area $\left[\mathrm{mm}^{2}\right]$ \\
\hline \hline 0.5 & 45 & 5.46 & 13.2 \\
0.5 & 45 & 5.59 & 12.8 \\
0.5 & 60 & 5.58 & 12.5 \\
0.5 & 60 & 6.23 & 13.8 \\
1.5 & 45 & 5.40 & 11.6 \\
1.5 & 60 & 5.51 & 0.0 \\
1.5 & 60 & 6.03 & 6.9 \\
3.0 & 45 & 5.53 & 0.0 \\
\hline
\end{tabular}

(b)

Figure 3: Compilation of experimental measurements of perforation area from tests conducted at Caltech's Small Particle Hypervelocity Impact Range (SPHIR) facility. Impactors are nylon $6 / 6$ right-cylinders $1.8 \mathrm{~mm}$ in diameter and with $L / D=1$. The target material is 6061-T6 aluminum. Data corresponds to commercially available plate thicknesses $h=0.5 \mathrm{~mm}, 1.5 \mathrm{~mm}$, and $3.0 \mathrm{~mm}$. a) $0^{\circ}$ obliquity. b) $45^{\circ}$ and $60^{\circ}$ obliquities.

Experiments were conducted at the Small Particle Hypervelocity Impact Range (SPHIR) Facility at Caltech [1, 35, 34]. This facility utilizes a twostage light gas gun with a $1.8 \mathrm{~mm}$ bore diameter launch tube and is capable of producing mass-dependent impact velocities ranging from 2 to $10 \mathrm{~km} / \mathrm{s}$ [20]. Nylon $6 / 6$ right-cylinders with a $1.8 \mathrm{~mm}$ diameter $(\mathrm{L} / \mathrm{D}=1)$ were used as impactors. The SPHIR facility is capable of accelerating the $5.5 \mathrm{mg}$ Nylon impactors to speeds between 5 and $7 \mathrm{~km} / \mathrm{s}$. Impact speeds were measured with a relative accuracy of $1.5 \%$ [34]. The target material selected was 6061-T6 aluminum. This extensively characterized material is inexpensive, readily available, and commonly used in aerospace structures. Impacted targets plates were $150 \mathrm{~mm} \times 150 \mathrm{~mm}$ and varied in thickness. Commercially available plate thicknesses of $0.5 \mathrm{~mm}, 1.5 \mathrm{~mm}$, and $3.0 \mathrm{~mm}$ were selected to provide a full spectrum of impactor size to plate thickness ratios. Impact obliquities of 0,45 , and 60 degrees with respect to plate-normal were selected to provide a wide range of expected target responses. Perforation area was defined as the area projected by a collimated light source through the target and onto a plane parallel to the original target back-surface. An 
Optimet MiniConoscan 3000 laser conoscope system was used to measure the perforation area of each impacted target plate, as reported in previous work [1]. The complete experimental data set obtained in support of the OUQ analysis is shown in Fig. 3.

\subsection{Material and computational model}

The uncertainty quantification protocol presented subsequently relies critically on the availability of a model that approximates the experimental data to within a quantified modeling error. The model used in the UQ analysis that follows is described in detail in [27], where a thorough experimental validation of the model is also presented. Here we briefly summarize the main features of the model and present selected comparisons with experiment for completeness.

In addition to transporting mass efficiently, a hypervelocity impact solver must account for complex contact, fracture and fragmentation phenomena. In this work, we use a computational model based on the Optimal-Transportation Mesh-Free (OTM) method of Li et al. [25, 26] extended to account for contact and fracture, $[43,38]$. The OTM method combines: i) optimal transportation concepts such as the Wasserstein distance [57] between successive mass densities in order to discretize the action integral in time; ii) Maximum-entropy (max-ent) meshfree interpolation [2] from a nodal-point set in order to avoid mesh entanglement and the need for continuous remeshing in simulations of unconstrained flows; and iii) material-point sampling $[25,26]$ in order to track the local state of material points and carry out complex constitutive updates.

The optimal-transportation approach to time discretization leads to geometrically exact updates of the local volumes and mass densities, as well as exact conservation properties including symplecticity, linear and angular momentum and energy [22]. Max-ent interpolation [2] offers the advantage of being mesh-free and entirely defined - essentially explicitly - by the current nodal-set positions. The mesh-free character of OTM confers it the ability to simulate unconstrained flows, be they solid, e. g., resulting from finite plasticity, or in the liquid or gaseous phase. A additional convenient feature of OTM, which is common to other material point methods [54] is that seizing contact is accounted for automatically, essentially for free. The relative ease with which OTM can handle complex contact configurations and collision sequences involving thousands of fragments in both convergent and divergent debris and ejecta flows is of paramount importance to the simulation of hypervelocity impact phenomena. 
A final necessary component of the material model concerns the simulation of material failure, fracture and fragmentation. Specifically, we simulate fracture by a variational material-point failure scheme known as eigenfracture $[43,38]$. In this scheme, the energy-release rate attendant to the failure of a material point is estimated by a local energy-averaging procedure, and material points are failed when the attendant energy-release rate exceeds the specific fracture energy of the material. Because the calculation of the effective energy-release rate at a material point is carried out within a local neighborhood of the point and it requires no explicit representation of the crack, the implementation of the material-point failure scheme is straightforward. The eigenfracture scheme is known to properly converge to Griffith fracture in the limit of vanishingly small mesh sizes [43].

Hypervelocity impact additionally sets in motion complex material behavior that needs to be carefully modeled in order for calculations to be predictive, in the sense of conforming accurately to experimental results. Such behavior includes extremely high deformations and strain rates, temperatures possibly resulting in solid-liquid-gas phase transitions, and complex fracture and fragmentation processes. Phase transitions are particularly prevalent in the nylon impactor, which vaporizes upon contact. These phenomena place exacting demands on solvers, which must be able to deal with solid-liquid-gas multiphase flows, and materials models, which must be predictive over a wide range of conditions.

We model the behavior of the aluminum 6061-T6 target plate within variational thermodynamic framework $[60,48]$ extended to the extreme conditions encountered in hypervelocity impact. The model employs equations of state (EoS) to describe the volumetric response in the high pressuretemperature regimes, accounts for thermoelasticity and thermoviscoplasticity, temperature dependent viscosity, and accounts for the partition of plastic work into stored and dissipated energy. The elastic deviatoric response is assumed to be quadratic in the elastic logarithmic strains, with isotropic elastic coefficients depending linearly on temperature and vanishing at the melting temperature. The volumetric material response in the high pressure and temperature regime is described by means of the SESAME equation of state $\left(\right.$ EoS) database [11]. A $J_{2}$-viscoplasticity model including power-law hardening, rate-sensitivity and thermal softening is used to characterize the plastic response of the aluminum target (cf., e. g., [9, 59]). We account for the volume dependence of the melting temperature, or melting line, through a modified Lindemann relation [49]. All deformations are assumed to be ostensibly adiabatic, and the conversion of dissipation, both plastic and viscous, into heat is accounted for through the variational model of Yang et 
al. $[60,48]$. We account for the temperature dependence of the viscosity of aluminum through Assael et al. [3] model. In calculations we determine the coefficients $a_{1}$ and $a_{2}$ based on both experimental data and results from molecular dynamics simulations performed using ReaxFF [56]. In particular, the molecular dynamics data allow us to extend the function $\mu(T)$ to high temperatures not available from the experimental data but relevant to hypervelocity impact.

In the hypervelocity impact tests under consideration, which entail impact velocities in the range of 5 to $10 \mathrm{~km} / \mathrm{s}$, the Nylon $6 / 6$ projectile vaporizes nearly instantaneously upon impact. We therefore model Nylon $6 / 6$ in its gas phase only. We specifically assume a two-sided adiabat, polytropic in compression and quadratic in tension. We determine the constants from Hugoniot data [30] and from molecular dynamics simulations performed using ReaxFF [56]. These additional ReaxFF data allow us to extend the range of the EoS to full range pressures relevant to hypervelocity impact, for which experimental data are not available in the literature. Experimental EoS data for Nylon 6/6 in tension also appear to be lacking in the open literature. Here again, we fill in this gap in the experimental record through ReaxFF molecular dynamics calculations. These calculations show that the material loses its bearing capacity entirely beyond a certain volumetric deformation. We fit this behavior by means of a quadratic function of the volumetric deformation followed by abrupt failure. Material failure is assumed to take place when the cohesive energy density (CED) of the Nylon $6 / 6$ is attained. We specifically assume an exponential temperature dependence of the cohesive energy density, with coefficients fitted to ReaxFF molecular dynamics calculations. Failed Nylon 6/6 material points are simply assumed to contribute no forces - but to retain their full mass - in the equations of motion.

\subsubsection{Assessment of modeling error}

Fig. 4 shows synthetic shadowgraphs at $0.9,1.7,2.5$ and $3.4 \mu \mathrm{s}$ from a OTM simulation of cylindrical Nylon $6 / 6$ projectiles of aspect ratio $L / D=1$ striking two Al6061 T6 plates of thickness 0.5 and $3.0 \mathrm{~mm}$ at velocities of $5.44 \mathrm{~km} / \mathrm{s}$ and $5.59 \mathrm{~km} / \mathrm{s}$, respectively, and zero obliquity. As may be seen from the figure, the nylon projectile is vaporized on contact. The perforation of the plate is accompanied by profuse fragmentation resulting in forward and backward debris ejecta. Interestingly, these debris ejecta are far from homogeneous and exhibit considerable structure, e. g., in the form of streamers. Fig. 5 shows a comparison of synthetic (OTM) and measured (SPHIR) shadowgraphs at $0.3 \mu \mathrm{s}, 1.2 \mu \mathrm{s}$ and $4.2 \mu \mathrm{s}$, for a Nylon $6 / 6$ cylin- 


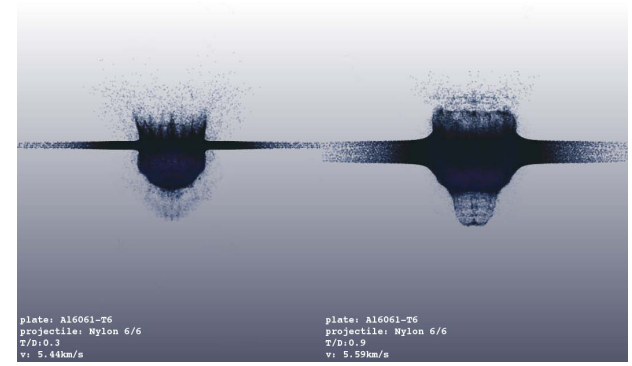

(a)

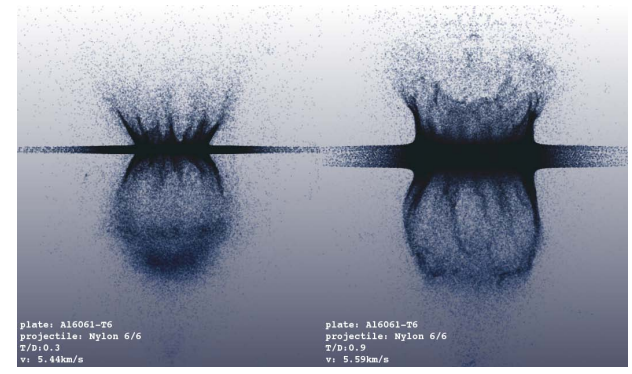

(c)

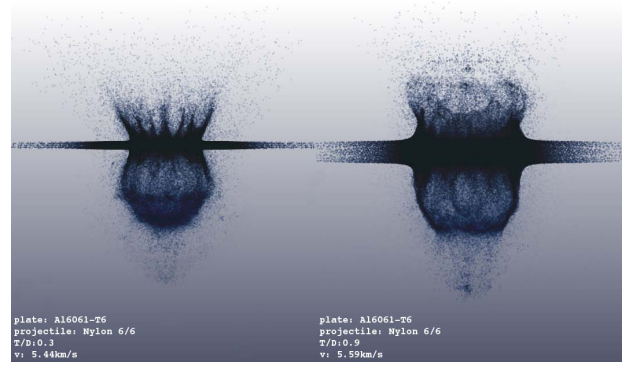

(b)

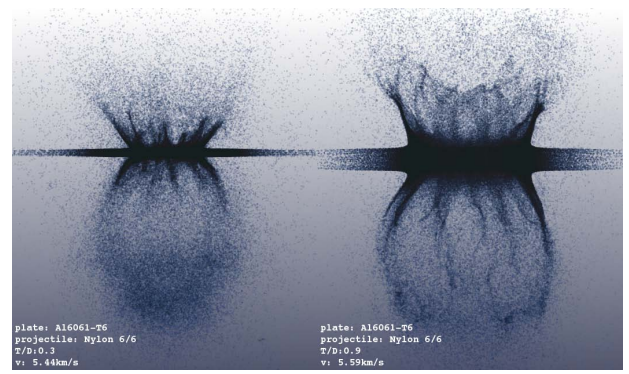

(d)

Figure 4: Snapshots at $0.9,1.7,2.5$ and $3.4 \mu$ s from an OTM calculation corresponding to cylindrical Nylon $6 / 6$ projectiles of aspect ratio $L / D=1$ striking two Al6061 T6 plates of thickness 0.5 and $3.0 \mathrm{~mm}$ at velocities of $5.44 \mathrm{~km} / \mathrm{s}$ and $5.59 \mathrm{~km} / \mathrm{s}$, respectively, and zero obliquity [27].
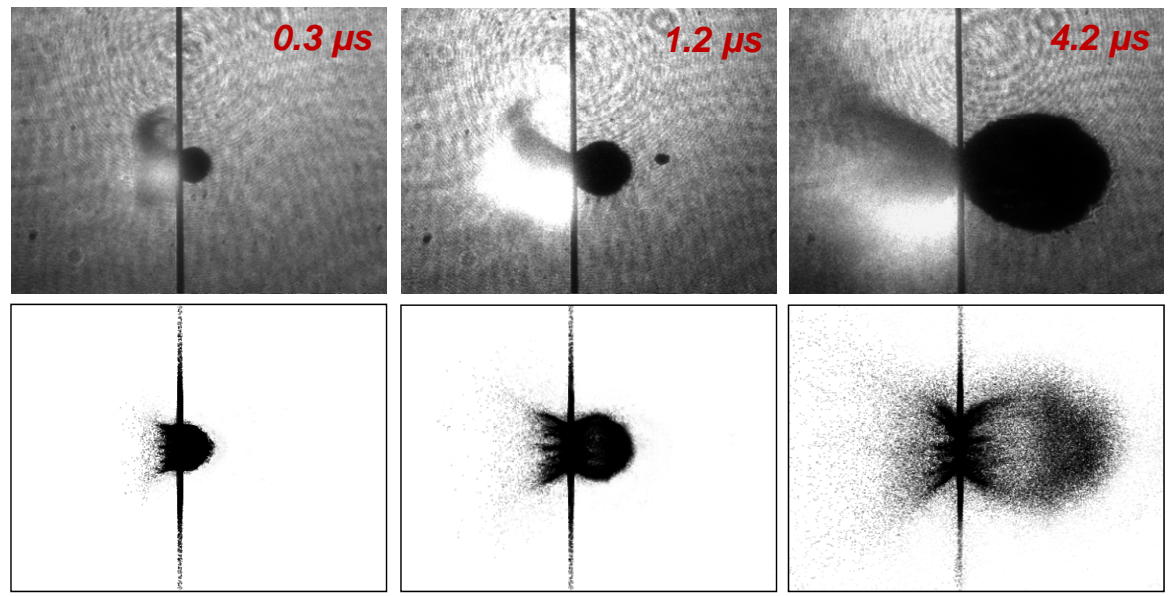

Figure 5: Comparison of experimentally measured (top) and calculated (bottom) shadowgraphs at $0.3 \mu \mathrm{s}, 1.2 \mu \mathrm{s}$ and $4.2 \mu \mathrm{s},[27]$. Nylon $6 / 6$ cylindrical impactor $(L / D=1)$, 6061-T6 Al target $\left(0.5 \mathrm{~mm}\right.$ thickness), impact velocity $5.84 \mathrm{~km} / \mathrm{s}, 0^{\circ}$ obliquity. 


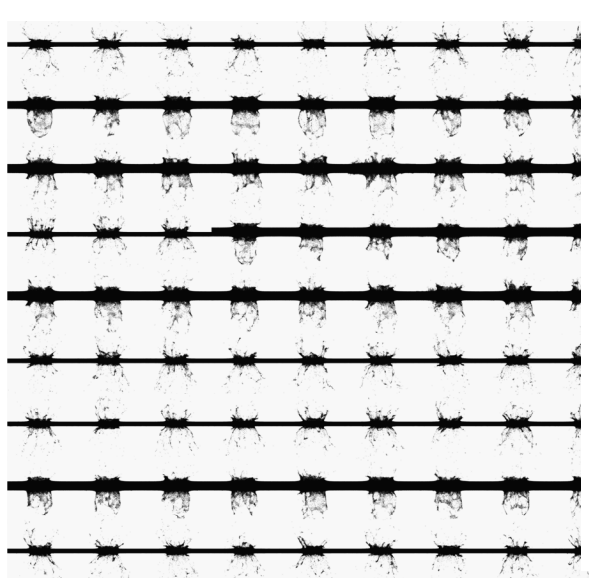

(a)

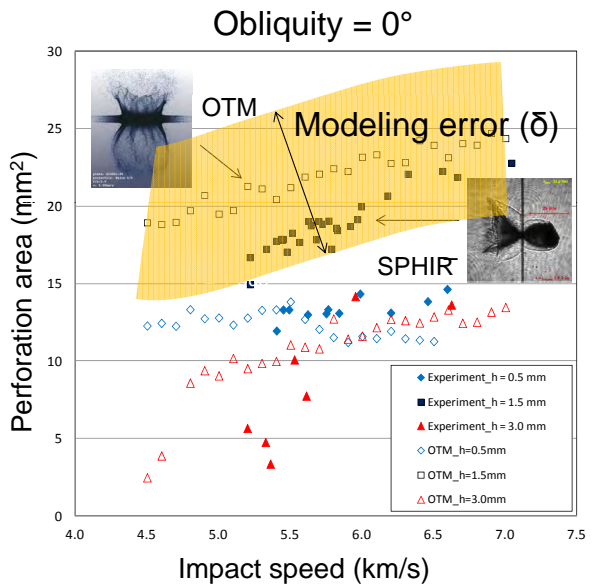

(b)

Figure 6: a) Part of a sequence of OTM calculations for various values of the input parameters (impact velocity, plate thickness, obliquity). b) Comparison of predicted and experimentally measured perforation areas and schematic of $d$-neighborhood of the model for a modeling error $\delta$. Each OTM point averages four runs with different yaw angles, which is an uncontrollable parameter in the experiments.

drical impactor $(\mathrm{L} / \mathrm{D}=1)$, a 6061-T6 $\mathrm{Al}$ target $0.5 \mathrm{~mm}$ in thickness, and impact velocity of $5.84 \mathrm{~km} / \mathrm{s}$ and $0^{\circ}$ obliquity. As may be seen from this comparison, the OTM solver and material model predict the observed overall progression and general structure of the experimental shadowgraphs. In general terms, the ability of the OTM solver to simulate the complex material behavior is remarkable, particularly in terms of its ability to resolve phase transitions and the complex fracture and fragmentation patterns that result from hypervelocity impact. Fig. 6a shows part of a sequence of OTM calculations with input parameters (impact velocity, plate thickness, obliquity) generated randomly over their range of definition as part of this paper's uncertainty quantification analysis. Each run uses of the order of one million material points to discretize plate and projectile and is performed using 512 mpi tasks in about 10 hours on a number of supercomputing platforms (hera, glory, mapache, moonlight, cab, chama) at the NNSA laboratories.

For the thinnest plates $(h=0.5 \mathrm{~mm}, h / D=0.3)$, the model predicts the perforation area to be ostensibly constant and independent of velocity in the impact-speed range of 6 and $7 \mathrm{~km} / \mathrm{s}$, in agreement with experiment. However, the calculated perforation area systematically underpredicts the experimental data. Effects not accounted for in the Nylon 6/6 model used 
in calculations, including ionization and plasma formation, are likely to contribute to this discrepancy. For the medium thickness plate $(h=1.5 \mathrm{~mm}$, $h / d=0.8)$ the reverse trend is observed, with the model overpredicting the perforation area. Here again, missing physics in the Nylon 6/6 model, such as radiation, are likely to account for the discrepancy. This trend is accentuated for the thicker plates $(h=3.0 \mathrm{~mm}, h / D=1.6)$, Fig. $6 \mathrm{~b}$. As may be seen from the figure, the OTM simulations consistently overestimate the perforation area, indicative of a systematic bias in the calculations. The modeling error (i. e. the discrepancy between the OTM simulation and corresponding experimental measurement) is largest in the lower range of impact velocities and narrows with increasing impact velocity, Fig. 6b. In particular, the OTM simulations underpredict the experimentally observed ballistic limit of 4.5 to $5.0 \mathrm{~km} / \mathrm{s}$.

\subsection{Uncertainty analysis}

The reduction theorem conveniently reduces the OUQ analysis to an optimization over a finite dimensional space of trial input parameters and their corresponding weights. In calculations, the evaluation of the objective function is accelerated by precomputing the OTM model on a regular grid over parameter space. We carry out the requisite optimizations using the a Differential Evolution [40,50] optimization algorithm implemented in the mystic framework $[32,33]$. We proceed to evaluate different scenarios illustrative of the range and scope of the legacy OUQ protocol.

\subsubsection{Model and legacy data only}

Our analysis employs the material and computational model $F_{M}: \mathcal{X} \rightarrow$ $\mathcal{Y}$ described in Section 3.2 in order to estimate the response of the system. We then use the distances (22) or (8) to define neighborhoods $\left\{d\left((X, F(X)), \Gamma_{F_{M}}\right) \leq\right.$ $\delta$ \} of width $\delta$ around the graph $\Gamma_{M}$ of the model, cf. Fig. $6 \mathrm{~b}$. In view of the cliff behavior of the system, we metrize the product space $\mathcal{X} \times \mathcal{Y}$ by means of the normalized Hausdorff distance

$$
d\left((x, y),\left(x^{\prime}, y^{\prime}\right)\right) \equiv \frac{\left|h-h^{\prime}\right|}{6 R_{h}}+\frac{\left|v-v^{\prime}\right|}{6 R_{v}}+\frac{\left|\alpha-\alpha^{\prime}\right|}{6 R_{\alpha}}+\frac{\left|y-y^{\prime}\right|}{2 R_{y}},
$$

when the the system is allowed to operate near the ballistic limit, and the supremum norm distance (8) otherwise. In (22), $R_{i}$ denotes the range of input variable $i$ and the weights are chosen so as to accord inputs and outputs equal importance.

Using Bernoulli trials, Section 2.3.2, and the experimental data described in Section 3.1, which contains $n=65$ data points, we then compute lower 


\begin{tabular}{r||r|r}
\hline model error $\delta$ & confidence $\eta=0.89$ & confidence $\eta=0.96$ \\
\hline $0.0378\left(3 \mathrm{~mm}^{2}\right)$ & 0.38 & 0.35 \\
$0.0503\left(4 \mathrm{~mm}^{2}\right)$ & 0.72 & 0.69 \\
$0.0629\left(5 \mathrm{~mm}^{2}\right)$ & 0.78 & 0.75 \\
$0.0881\left(7 \mathrm{~mm}^{2}\right)$ & 0.86 & 0.83 \\
$0.1133\left(9 \mathrm{~mm}^{2}\right)$ & 0.88 & 0.85 \\
$0.1384\left(11 \mathrm{~mm}^{2}\right)$ & 0.88 & 0.85 \\
\hline
\end{tabular}

Table 2: Optimal lower bound on the probability $p_{\delta}=\mathbb{P}\left\{\left|Y-F_{M}(X)\right| \leq \delta\right\}$ for two confidence levels $\eta$.

bounds on the probability $p_{\delta}$ that the actual response function of the system lie in the neighborhood $\left\{d\left((X, F(X)), \Gamma_{F_{M}}\right) \leq \delta\right\}$ with prespecified confidence $\eta \equiv 1-\epsilon^{\prime}$. The resulting optimal probability lower bounds are collected in Table 2. This table collects optimal lower bounds on the probability $p_{\delta}$ corresponding to increasing allowances for modeling error $\delta$ and for two confidence levels $\eta=0.89$ and 0.96 . This table may be regarded as a quantitative assessment of modeling error, or model validation, in so far as it quantifies the confidence that may be had on the actual response of the system being within a certain distance of the model. Once the fidelity of the model is established by this means, the model can then stand in for the experimental data in the UQ analysis and probabilities of outcomes computed directly from the model with rigorously quantified uncertainties.

Thus, suppose, by way of example, that wish to determine a neighborhood around the graph $\Gamma_{F_{M}}$ of the model such that the actual response of the system be in the neighborhood with probability $p_{\delta}$ at least 0.85 , and we wish to have a confidence at least $1-\epsilon^{\prime}=0.96$ that that probability is indeed a lower bound. Then, from Table 2 it follows that we have to allow for a modeling error $\delta=0.1133$, corresponding to a perforation area error of the order of $9 \mathrm{~mm}^{2}$. If, by way of contrast, a confidence $1-\epsilon^{\prime}=0.89$ in the certification assessment suffices, then a neighborhood of width $\delta=0881$ around the model, corresponding roughly to a perforation area error of the order of $7 \mathrm{~mm}^{2}$, would guarantee that the actual response of the system be in the neighborhood with probability $p_{\delta}$ at least 0.86 , in turn resulting in a great likelihood that the system is certifiable. These trade-offs between availability of experimental data, model uncertainty, certifiability and confidence in the certification assessment are noteworthy.

If the only information available about the system consists of legacy 
data, then the admissible set (7) for OUQ is

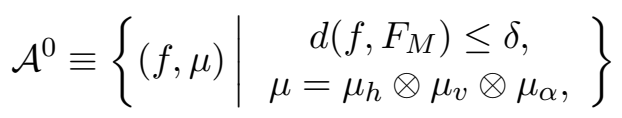

with probability $p_{\delta}$. The reduction theorem, Section 2.2 , then gives the reduced admissible set

$$
\mathcal{A}_{\Delta}^{0} \equiv\left\{\begin{array}{l|l}
(f, \mu) \in \mathcal{A}^{0} \mid \begin{array}{c}
\text { for } k \in\{h, v, \alpha\}: \\
\mu_{k}=\delta_{x_{k}}, x_{k} \in \mathcal{X}_{k} .
\end{array}
\end{array}\right\}
$$

Evidently, in this case OUQ reduces to worst-case analysis, consisting of finding the input parameters $\left(x_{k}, k \in\{h, v, \alpha\}\right)$ resulting in the worst performance within the admissible set $\left\{d\left((X, Y), F_{M}\right) \leq \delta\right\}$. The optimal probability-of-failure upper bound, given the available information, is then 1 if the worst-case performance is below the admissible threshold for safe operation of the system, and $1-p_{\delta}$, with confidence $\eta$, otherwise.

For instance, suppose that we confine attention to the thinnest plates, $h=0.5 \mathrm{~mm}$, and normal incidence, $\alpha=0^{\circ}$. Allow for a modeling error $\delta=11 \mathrm{~mm}^{2}$. Then, a direct examination of Fig. $6 \mathrm{~b}$ shows that, even in the worst case, corresponding to the slowest impact velocity $v=4.5 \mathrm{~km} / \mathrm{s}$, the target plate is always perforated in laboratory tests. Thus, in this scenario we read from Table 2 that the lethality of the projectile can be established with probability 0.88 and confidence 0.89 , or with probability 0.85 and confidence 0.96 . We note that a certain probability of failure remains, despite the plate always being perforated in experiments, owing to overall uncertainties in the model, the limited size of the experimental sample and the extent of experimental scatter thereof. If, by way of contrast, we consider the thickest plates, $h=3.0 \mathrm{~mm}$, at normal incidence, $\alpha=0^{\circ}$, again with reference to Fig. 6b, we observe a lack of perforation in the worst-case scenario, which again corresponds to the slowest impact velocity $v=4.5 \mathrm{~km} / \mathrm{s}$, beyond distances from the model of, say $\delta=3 \mathrm{~mm}^{2}$. Thus, in this case it follows from and Table 2 that the lethality of the projectile can only be established with probability 0.38 and confidence 0.89 , or with probability 0.35 and confidence 0.96 .

\subsubsection{Moment constraints}

We additionally illustrate the ability of the OUQ protocol to account for moment constraints by means of two examples, namely, bounds on the perforation area and mean constraints on the input variables. These moment constraints arise when the distribution of inputs and/or outputs recorded 
during the operation of the system is known to be biased within the operating range. In such cases, the additional information can be used to narrow the admissible set $\mathcal{A}$ and sharpen the OUQ analysis.

In practice, moment bounds $(m, M)$ and $\left(m_{k}, M_{k}\right), k \in\{h, v, \alpha\}$, can be determined probabilistically using the method described in Section 2.3.3. By this means, we find from the data set described in Section 3.1, that, with probability at least $\gamma \equiv 0.9$, the input and output parameter means lie within the intervals: $\mathbb{E}_{\mu}[g(h, v, \alpha)] \in\left[8.79 \mathrm{~mm}^{2}, 20.85 \mathrm{~mm}^{2}\right] ; \mathbb{E}_{\mu_{h}}[h] \in$ $[1.06 \mathrm{~mm}, 1.82 \mathrm{~mm}] ; \mathbb{E}_{\mu_{v}}[v] \in\left[5.37 \mathrm{~km} \cdot \mathrm{s}^{-1}, 6.13 \mathrm{~km} \cdot \mathrm{s}^{-1}\right] ; \mathbb{E}_{\mu_{\alpha}}[\alpha] \in\left[0^{\circ}, 15.57^{\circ}\right]$. These bounds can now be introduced in the admissible sets (25) and (29) for purposes of OUQ analysis.

Mean constraint on outputs. Suppose that we have additional information on the response of the system in the form of upper and lower bounds, $M$ and $m$ respectively on the mean response $\mathbb{E}_{\mu}\{f(h, v, \alpha)\}$ of the system. The corresponding admissible set is, then,

$$
\mathcal{A}^{1} \equiv\left\{\begin{array}{l|c}
d(f, \mu) \mid \begin{array}{c}
d\left(F_{M}\right) \leq \delta \\
\mu=\mu_{h} \otimes \mu_{v} \otimes \mu_{\alpha}, \\
m \leq \mathbb{E}_{\mu}\{f(h, v, \alpha)\} \leq M .
\end{array}
\end{array}\right\}
$$

In this case, the reduction theorem, Section 2.2, then gives the reduced admissible set as

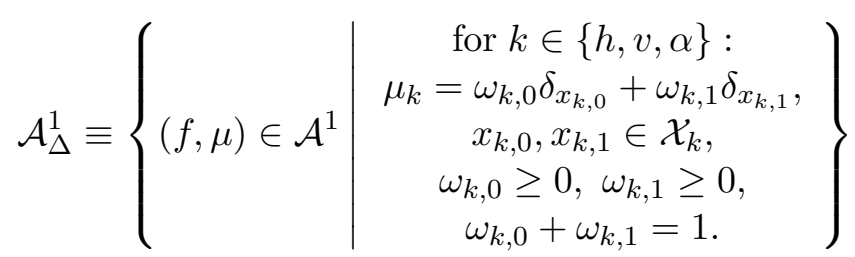

Evidently, in this scenario OUQ is no longer a worst-case analysis, as it plays two possible sets of admissible inputs, $\left(x_{k, 0}, k \in\{h, v, \alpha\}\right)$ and $\left(x_{k, 1}, k \in\right.$ $\{h, v, \alpha\}$ ), against each other with probabilities $\omega_{k, 0}$ and $\omega_{k, 1}$, respectively.

Fig. 7 shows the results of an OUQ analysis under the scenario just described. The results are presented as OUQ least-upper-bounds $\mathcal{U}\left(\theta \mid \mathcal{A}^{(1)}\right)$ on the probability $\mathbb{P}\{F(X) \leq \theta\}$ that the perforation area $F(X)$ be less that $\theta$ with confidence $\eta=0.89$, using the uniform distance, Fig. $7 \mathrm{a}$, and the Hausdorff distance, Fig. 7b, to define confidence intervals around the model. If, for instance, the intent of the analysis is to certify the lethality of the projectile, then the system is certified with confidence $\eta$ if $\theta$ is the minimum acceptable perforation area such that $\mathcal{U}\left(0 \mid \mathcal{A}^{(1)}\right) \leq \epsilon$, where $\epsilon$ is the failure tolerance. 


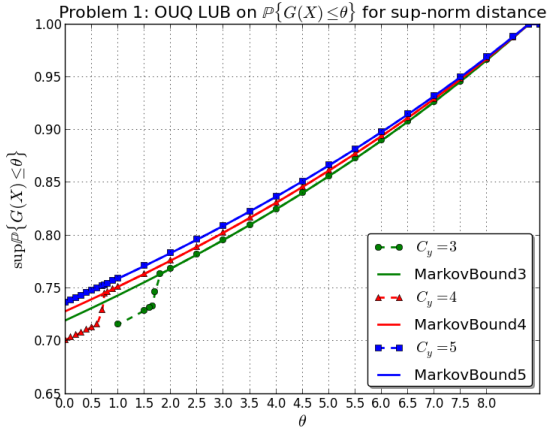

(a)

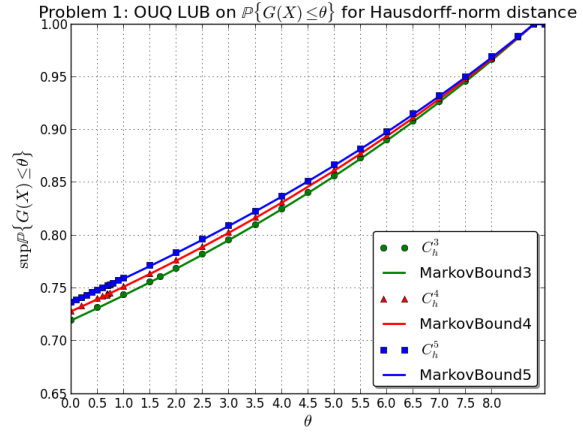

(b)

Figure 7: OUQ least-upper-bound $\mathcal{U}\left(\mathcal{A}^{1}\right)$ on $\mathbb{P}\{F(X) \leq \theta\}$ for the problem with mean constraints on outputs. Results shown for different neighborhood sizes $\delta$ around the model and for confidence $\eta=0.89$. Markov bounds are represented with dash lines. a) Supremum-norm distance. b) Hausdorff distance.

As expected smaller values of $\delta$, corresponding to tighter confidence intervals around the model, result in smaller least-upper-bounds on probabilities of outcomes. Fig. 7 also reveals that optimal bounds are insensitive to the accuracy of the model. Thus, for instance, the differences between the optimal bounds for $\delta=3 \mathrm{~mm}^{2}$ and $\delta=5 \mathrm{~mm}^{2}$ is relatively small. This lack of sensitivity owes to the fact that information on the probability distribution of input variables is limited to a mean constraint on the response function. Also shown in Fig. 7 is the Markov bound [10]

$$
\mathbb{P}\{F(h, \alpha, v) \leq \theta\} \leq \frac{y_{\max }-m}{y_{\max }-\theta}
$$

where

$$
y_{\max }=\sup _{(h, v, \alpha) \in \mathcal{X}} F_{M}(h, v, \alpha)+C
$$

with maximizer at $(h, v, \alpha)=\left(1.5 \mathrm{~mm}, 6.9 \mathrm{~km} \cdot \mathrm{s}^{-1}, 60^{\circ}\right)$, and $m$ is the lower bound in the feasible set described in equation 25 . The close agreement between the OUQ bounds and the Markov bound is remarkable.

Another noteworthy observation pertaining to the OUQ analysis is that the majority of the Dirac masses on input variables are observed to merge, or collapse, during the execution of the numerical optimization algorithm. More precisely, the marginals $\mu_{v}$ and $\mu_{\alpha}$ of the measure $\mu \equiv \mu_{h} \otimes \mu_{v} \otimes \mu_{\alpha}$ collapse to one single Dirac. Once this dimensional collapse phenomenon 
is uncovered, even approximately, the calculation of $\mathcal{U}(\theta \mid \mathcal{A})$ can be greatly accelerated by reducing the number of marginal support points accordingly.

Mean constraints on inputs. Suppose now that we have additional information on the distribution of the inputs that the system is likely to operate under in the form of upper and lower bounds, $M_{k}$ and $m_{k}$, respectively, on the mean values $\mathbb{E}_{\mu_{k}}\left[x_{k}\right], k \in\{h, v, \alpha\}$, of the inputs. The corresponding admissible set is, then,

$$
\mathcal{A}^{2} \equiv\left\{(f, \mu) \mid \begin{array}{c}
d\left(f, F_{M}\right) \leq \delta \\
\mu=\mu_{h} \otimes \mu_{v} \otimes \mu_{\alpha} \\
\text { for } k \in\{h, v, \alpha\} \\
m_{k} \leq \mathbb{E}_{\mu_{k}}\left[x_{k}\right] \leq M_{k}
\end{array}\right\}
$$

which after reduction becomes,

$$
\mathcal{A}_{\Delta}^{2} \equiv\left\{\begin{array}{l|c}
\text { for } k \in\{h, v, \alpha\}: \\
\mu_{k}=\omega_{k, 0} \delta_{x_{k, 0}}+\omega_{k, 1} \delta_{x_{k, 1}}, \\
x_{k, 0}, x_{k, 1} \in \mathcal{A}_{k} \\
\omega_{k, 0} \geq 0, \omega_{k, 1} \geq 0 \\
\omega_{k, 0}+\omega_{k, 1}=1 .
\end{array}\right\}
$$

We note that, in this scenario, the theorems of [37] lead to the same reduced admissible set as in the preceding scenario, despite now having three moment constraints, one on each input parameter $(h, v, \alpha)$, instead of one constraint on the output. Indeed, one moment constraint on output increases by one the number of required Dirac masses on each input parameter. By contrast, one moment constraint on one input parameter only increases by one the number of required Dirac masses for that parameter.

Fig. 8 shows the results of an OUQ analysis under the scenario just described. The discontinuity at $y=0.53$ corresponds to the activation of a constraint, i e., to a constraint changing from non-active, or non-informative, to active, or informative. Again, smaller values of $\delta$, corresponding to tighter confidence intervals around the model, result in smaller least-upper-bounds on probabilities of outcomes. By contrast, in this scenario the optimal bounds show significant sensitivity to the accuracy of the model, or, more precisely, to the width $\delta$ of the confidence intervals. This enhanced sensitivity owes to the fact that, whereas a mean constraint on the quantity of interest remains informative with an inaccurate model, mean constraints on input variables carry no information about the quantity of interest without model accuracy. The sensitive dependence of the optimal bounds with respect to the choice of confidence interval raises the question of how the 


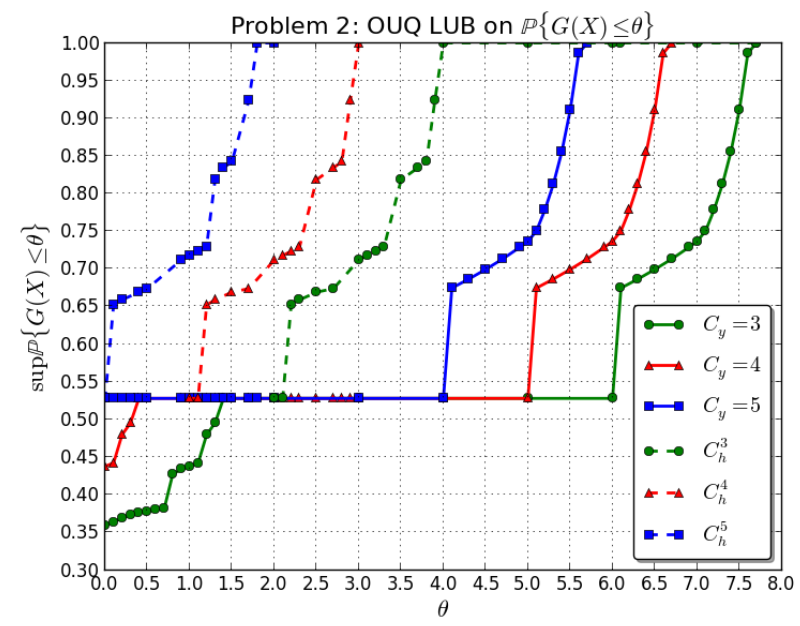

Figure 8: OUQ least-upper-bound $\mathcal{U}\left(\mathcal{A}^{2}\right)$ on $\mathbb{P}\{F(X) \leq \theta\}$ for the problem with mean constraints on inputs. Results shown for different neighborhood sizes $\delta$ around the model and for confidence $\eta=0.89$. Solid lines: Uniform distance. Dash lines: Hausdorff distance.

available information can be best utilized in a OUQ analysis in order to obtain the sharpest possible bounds. However, this problem is of great complexity and beyond the scope of the present work.

\subsection{Computational costs}

To conclude this section, we outline the optimization method used to solve the OUQ problems posed above, and the approximate costs. At a high level, the OUQ problem is expressed as an optimization problem with respect to Python objects that represent the discrete measures and functions (input-output pairs), or scenarios, that are the mathematical optimization variables, as in earlier OUQ studies such as [52]. These Python objects are converted into purely parametric representations, and the parametric problem is solved using the Differential Evolution (DE) algorithm of Price et al. [50, 39, 40]. Within this framework, while the human interface 'understands' probability-theoretic terms such as probabilities, expectations, and so on, the optimization problem that the DE algorithm handles is 'blind' to the probability-theoretic interpretation of the parameters that it manipulates.

OUQ seeks to determine extreme values of deviation probabilities of the form $(F, \mu) \mapsto \mathbb{P}_{\mu}\{F(X) \leq \theta\}$, i. e., lower and upper cumulative distribution 
functions, or CDF envelopes. Conveniently, the calculations corresponding to different values of the threshold $\theta$ provide useful cross-validation information about each other. For example, since both the minimum and maximum CDFs are increasing functions of $\theta$, if the numerical results do not have this property, then it is clear that an error, or, at least, an insufficiently resolved calculation, has occurred. In general, the CDF envelopes are observed to typically be piecewise smooth with occasional kinks or jump discontinuities: this heuristics also guides the acceptance or further investigation of the numerical optimization results for particular values of $\theta$.

The performance of the DE algorithm is principally controlled by the population size (npop)and the convergence/termination criterion. Naturally, a larger value for npop yields more extensive exploration of the optimization space and hence more robust solutions, but at an increased computational cost. For the calculations in this paper, npop was typically set to 40 or 60 . The convergence criterion used was a change-over-generations criterion, in which the optimization was terminated when no improvement of the objective function value greater than $10^{-6}$ is observed over ngen consecutive generations; typical values of ngen ranged from 20 (used to get a very rough understanding of the results) to 400 (for more fully resolved values, especially for $\theta$ near the kinks and jump discontinuities mentioned in the previous paragraph). A 'gold standard' optimization for a single $\theta$ value with npop $=60$ and ngen $=400$ would take approximately 8 core-hours; lower-fidelity optimizations, which to a large extent are sufficient for interpolating between higher-fidelity OUQ optimizations, were correspondingly faster.

\section{Comparison to other UQ approaches}

UQ is an umbrella term that encompasses a broad spectrum of methods. It is therefore of interest to contrast the legacy OUQ protocol to other existing methods.

Monte Carlo strategies. Monte Carlo strategies (cf., e. g., [28]) are simple to implement and do not require prior information other than i.i.d. samples. However, Monte Carlo methods may require a large number of independent samples, which is a severe limitation for the certification of systems whose failure is controlled by rare events. For instance, the maximum probability of a catastrophic event per hour of flight $\epsilon=10^{-9}$ of the aviation industry would $[47,8]$ necessitate $O\left(10^{18}\right)$ samples, a truly staggering number. In order to reduce the number of required samples, additional information on the system can be included in Monte Carlo methods via importance and 
weighted sampling [28]. However, these options are not available when only a fixed legacy data set is in existence.

Stochastic expansion methods. If the response function of the system of interest is regular enough and can be sampled at pre-determined points, and if the input variables have known distributions, then stochastic expansion methods $[19,18,58,4,16,14]$ can reduce the number of required samples even further, depending on the regularity of the response function, provided that the dimension of input variables is not too high $[55,7]$. Often, however, the response function is discontinuous or nearly discontinuous, e. g., in systems exhibiting cliff behavior, and, therefore, lacks the requisite regularity. In addition, the possibility of sampling at pre-determined points is not available when only a fixed legacy data set is accessible.

Quasi-Monte Carlo Methods. The number of required samples can also be reduced to $\frac{1}{\epsilon}\left(\log \frac{1}{\epsilon}\right)^{d}$, where $\epsilon$ is the probability-of-failure tolerance and $d$ is the dimension of input variables, using Quasi-Monte Carlo Methods. We refer in particular to the Koksma-Hlawka inequality [36], to [46] for multiple integration based on lattice rules and to [45] for a recent review. We observe that these methods require regularity of the response function and the possibility of sampling it at pre-determined points, an option that is not always available. Furthermore, the number of required samples blows-up at an exponential rate with the dimension $d$ of the input variables.

Sensitivity analysis. The quantification of variations in the response function corresponding to variations in input parameters, commonly referred to as sensitivity analysis [41, 42], allows for the identification of critical input parameters. Single parameter oscillations of the response function can also be seen as a form of non-linear sensitivity analysis leading to bounds on probabilities of failure via concentration inequalities $[31,29,53]$. However, in cases in which the response function is not differentiable, or has very large localized derivatives, in which case linear sensitivity analysis breaks down. In addition, the formulation of rigorous probability bounds often requires large-variation nonlinear sensitivity analysis [31, 29, 53], and standard linear sensitivity analysis does not suffice. Finally, sensitivity analysis pertains strictly to models of the response function. Since such models are bound to be inaccurate and uncertain, in order to achieve certification the model accuracy and uncertainty needs to be carefully assessed with the aid of data.

Bayesian inference. If the information about the system is expressed probabilistically through a prior, i. e., an a priori measure of probability, on the set possible scenarios, then Bayesian inference $[24,6]$ can be used to estimate the system response using the corresponding posterior proba- 
bility measure. Bayesian inference is a powerful method for incorporating information obtained from sampled data through Bayes' rule. For complex systems, the computation of posterior probabilities has become increasingly tractable due to advances in high-performance computing. We refer to [51] for a (recent) general Gaussian framework for Bayesian inverse problems and to [5] for a rigorous UQ framework based on probability logic with Bayesian updating. However, often priors are not available or their choice involves some degree of arbitrariness that is incompatible with the certification of systems whose failure is controlled by rare events. Thus, priors may become asymptotically irrelevant, in the limit of large data sets, and, for small probability-of-failure tolerances, the number of required samples can be of the same order as in Monte-Carlo methods [44]. In addition, when unknown parameters are estimated using priors and sampled data, it is important to observe that the convergence of the Bayesian method may fail if number of possible outcomes is not finite $[12,13]$.

Optimization and Anti-Optimization. The use of structural optimization to produce an optimal design given the deterministic worst-case scenario is referred to as Optimization and Anti-Optimization [17], also as critical excitation in seismic engineering [15]. The main difference between OUQ and Anti-optimization lies in the fact that the former is based on an optimization over admissible functions and input probability measures, whereas the latter involves an optimization over response functions only. Because of its robustness, many engineers have adopted the deterministic worst-case scenario approach to UQ (we refer to Chapter 10 of [17] for a lucid account), especially when high reliability is required. It is noted in [17] that "probabilistic reliability studies involve assumptions on the probability densities, whose knowledge regarding relevant input quantities is central". In addition, methods based on deterministic worst-case scenarios are oftentimes "too pessimistic to be practical" $[15,17]$. OUQ sidesteps these limitations of worst-case UQ by requiring limited knowledge of the probabilities of inputs only, and by accounting for the response of the system over its entire range of operation.

The preceding brief review reveals that the problem of formulating and computing rigorous upper and lower bounds on the probability of outcomes of complex systems in a manner that 'folds in' all the information available on the operating conditions and the response of the system in an effective manner remains an open challenge and that the existing methods often lack the necessary scope or eschew some of the central difficulties. In this context, OUQ may be regarded as a direct abstract formulation of the problem of optimal upper and lower bounds on probabilities of outcomes in which 
the information about the system provides optimality constraints. OUQ also provides a computational scheme for evaluating the optimal probability bounds of interest in a number of scenarios of practical interest, including the scenario contemplated in this work, namely, systems whose response is characterized by means of an empirical model and legacy data.

\section{Summary and discussion}

We have presented an Optimal Uncertainty Quantification (OUQ) protocol for systems that are characterized by an existing physics-based model and for which only legacy data is available, i. e., no additional experimental testing of the system is possible. Specifically, the OUQ strategy developed in this work consists of using the legacy data to establish, in a probabilistic sense, the level of error of the model, or modeling error, and to subsequently use the validated model as a basis for the determination of probabilities of outcomes. The quantification of modeling uncertainty specifically establishes, to a specified confidence, the probability that the actual response of the system lie within a certain distance of the model. Once the extent of model uncertainty has been established in this manner, the model can be conveniently used to stand in for the actual or empirical response of the system in order to compute probabilities of outcomes. To this, we resort to the reduction theorem of Owhadi et al. [37] in order to reduce the computation of optimal upper and lower bounds on probabilities of outcomes to a finite-dimensional optimization problem.

We have illustrated the resulting UQ protocol by means of an application concerned with the response to hypervelocity impact of 6061-T6 Aluminum plates by Nylon 6/6 impactors at impact velocities in the range of $5-7 \mathrm{~km} / \mathrm{s}$. The system under consideration is characterized by three input parameters, namely, impact velocity, plate thickness, and obliquity, and the outcome of interest is the perforation area. The resulting UQ problem concerns, therefore, the determination of bounds on perforation area probabilities when the system is allowed to operate over a certain range of the input parameters. Experimental data in support of the UQ analysis is obtained at Caltech's Small Particle Hypervelocity Impact Range (SPHIR) [1, 35, 34]. Specifically, a legacy data set is generated from 65 hypervelocity shots providing coverage over the range of inputs. In addition, we employ physics-based, phaseaware models of 6061-T6 Al and Nylon 6/6 in conjunction with the Optimal Transportation Meshfree (OTM) [25, 26] Lagrangian solver, which includes fragmentation and contact capability, in order to model the hypervelocity tests. Finally, optimal upper bounds on perforation-area probabilities, with 
or without additional a priori information in the form of input and output moment constraints, are obtained using the mystic framework $[32,33]$ in order to carry out the requisite optimization calculations. Overall, the ability of OUQ to process diverse information on the system and its ability to supply rigorous bounds on system performance under realistic - and less than ideal-scenarios is remarkable.

We emphasize that the OUQ framework, as applied in this work, makes quantitatively explicit the role of the model, the metrization of the inputoutput product space, and the trade-off between the width of the model neighborhood and certification confidence. Thus, the larger the model neighborhood, the more failure configurations in contains, thus leading to a conservative prediction of a high probability of failure, but also the more experimental data points it also contains, thus leading to high confidence in the certification. Conversely, the smaller the model neighbourhood, the fewer failure configurations in contains, thus leading to a bold prediction of a low probability of failure, but the fewer data point it also contains, leading to low confidence in the certification. This tradeoff may be regarded as being loosely analogous to the tradeoff between Type I and Type II errors in classical statistical testing. The definition of acceptable performance and confidence levels is a meta-judgement, or 'policy decision', that is made outside the scope of the UQ framework. However, the internal consistency of the UQ framework and explicit quantitative inter-relations between performance certification and confidence themselves provide confidence in the procedure for decision-makers, and are mathematically interesting properties worthy of development in future works.

A number of underlying assumptions and limitations of the proposed OUQ protocol merit careful discussion. The reliance on sample legacy data to characterize the system confers OUQ a probabilistic nature and any assessment of the system can only be carried out in terms of confidence intervals. It is interesting to note that, under such conditions, the role of the physics-based model is somewhat limited and for the most part limited to organizing and interpolating the data. The use of a validated physics-based model as stand-in for the legacy data in OUQ calculations is also mainly a matter of convenience, especially in the absence of a priori information on the accuracy of the model. We note, however, that such convenience comes at a price, is so far as the model uncertainty compounds other uncertainties and ultimately reduces the certifiability of the system. In this work we specifically quantify model uncertainty based on Bernoulli trials, Section 2.3.2, under the assumption that the legacy data consists of independent and identically distributed (i.i.d.) experimental realizations of samples 
from the system. In practice, the sampling of experimental conditions may originate from extraneous considerations as part of the design of the experimental campaign and may not necessarily reflect the actual distribution of operating conditions once the system is deployed in the field. A related fundamental theoretical question concerns the formulation of optimal statistical estimators that extract as much information as possible from the available sample data. Recall that a statistical estimator is a function of the sample data designed to infer an unknown quantity. In the present context, we particularly wish to use legacy data to make inferences regarding the unknown response function of the system. The development of optimal statistical estimators is a challenging problem in probability as it requires the solution of optimization problems whose variables are measures over spaces of measures and functions, in contrast to present OUQ in which the optimization variables are measures and functions. However, reduction theorems such as presented in [37] can in principle reduce the computation of the optimal statistical estimators to a finite-dimensional optimization problem. The OUQ framework can also be generalized by allowing for information in the form of - possibly incompletely specified - priors and likelihoods, thus subsuming traditional approaches to the handling of sample data based on Bayesian learning. These and other extensions of the OUQ paradigm suggest additional worthwhile avenues for future research.

\section{Acknowledgments}

The authors gratefully acknowledge the support of the Department of Energy National Nuclear Security Administration under Award Number DE-FC52-08NA28613 through Caltech's ASC/PSAAP Center for the Predictive Modeling and Simulation of High Energy Density Dynamic Response of Materials.

[1] Adams, M., Lashgari, A., Li, B., Mc Kerns, M., Mihaly, J., Ortiz, M., Owhadi, H., Rosakis, A. J., Stalzer, M., Sullivan, T. J., 2012. Rigorous model-based uncertainty quantification with application to terminal ballistics. Part II. Systems with uncontrollable inputs and large scatter. Journal of the Mechanics and Physics of Solids 60 (5), 1002-1019.

[2] Arroyo, M., Ortiz, M., 2006. Local maximum-entropy approximation schemes: a seamless bridge between finite elements and meshfree methods. International Journal for Numerical Methods in Engineering 65 (13), 2167-2202. 
[3] Assael, M. J., Kakosimos, K., Banish, R. M., Brillo, J., Egry, I., Brooks, R., Quested, P. N., Mills, K. C., Nagashima, A., Sato, Y., Wakeham, W. A., 2006. Reference Data for the Density and Viscosity of Liquid Aluminum and Liquid Iron. Journal of Physical and Chemical Reference Data 35 (1), 285-300.

[4] Babuška, I., Nobile, F., Tempone, R., 2010. A stochastic collocation method for elliptic partial differential equations with random input data. SIAM Rev. 52 (2), 317-355.

URL http://dx.doi.org/10.1137/100786356

[5] Beck, J. L., 2010. Bayesian system identification based on probability logic. Struct. Control Health Monit. 17, 825-847.

[6] Beck, J. L., Katafygiotis, L. S., 1998. Updating models and their uncertainties: Bayesian statistical framework. Journal of Engineering Mechanics 124 (4), 455-461.

[7] Bieri, M., Schwab, C., 2009. Sparse high order FEM for elliptic sPDEs. Comput. Methods Appl. Mech. Engrg. 198 (13-14), 1149-1170.

URL http://dx.doi.org/10.1016/j.cma.2008.08.019

[8] Boeing, July 2010. Statistical summary of commercial jet airplane accidents worldwide operations 1959 - 2009. Tech. rep., Aviation Safety Boeing Commercial Airplanes, Seattle, Washington 98124-2207, U.S.A.

[9] Camacho, G. T., Ortiz, M., 1997. Adaptive Lagrangian modelling of ballistic penetration of metallic targets. Computer methods in applied mechanics and engineering 142 (3-4), 269-301.

[10] Carlen, E., Kusuoka, S., Stroock, D. W., 1987. Upper bounds for symmetric markov transition functions. Ann. Inst. Henri Poincaré 2, 245287.

[11] Crockett, S. D., T-1, 2004. Los Alamos National Laboratory Report No. LA-UR-04-6442, material: SESAME 003720. Tech. rep., Los Alamos National Laboratory.

[12] Diaconis, P., Freedman, D., 1986. On the consistency of Bayes estimates. Ann. Statist. 14 (1), 1-67, with a discussion and a rejoinder by the authors.

URL http://dx.doi.org/10.1214/aos/1176349830 
[13] Diaconis, P. W., Freedman, D., 1998. Consistency of Bayes estimates for nonparametric regression: normal theory. Bernoulli 4 (4), 411-444. URL http://dx.doi.org/10.2307/3318659

[14] Doostan, A., Owhadi, H., 2011. A non-adapted sparse approximation of PDEs with stochastic inputs 230 (8), 3015-3034.

[15] Drenick, R. F., 1973. Aseismic design by way of critical excitation. J. Eng. Mech. Div., Am. Soc. Civ. Eng. 99 (4), 649-667.

[16] Eldred, I. H., Webster, C. G., Constantine, P. G., 2008. Design under uncertainty employing stochastic expansion methods. American Institute of Aeronautics and Astronautics Paper 2008-6001.

[17] Elishakoff, I., Ohsaki, M., 2010. Optimization and Anti-Optimization of Structures Under Uncertainty. World Scientific, London.

[18] Ghanem, R., 1999. Ingredients for a general purpose stochastic finite elements implementation. Comput. Methods Appl. Mech. Engrg. 168 (14), 19-34.

URL http://dx.doi.org/10.1016/S0045-7825(98)00106-6

[19] Ghanem, R., Dham, S., 1998. Stochastic finite element analysis for multiphase flow in heterogeneous porous media. Transp. Porous Media $32(3), 239-262$.

URL http://dx.doi.org/10.1023/A:1006514109327

[20] Grosch, D. J., Riegel, J. P., 1993. Development and optimization of a "micro" two-stage light-gas gun. International Journal of Impact Engineering $14(1-4), 315-324$.

[21] Hoeffding, W., 1963. Probability inequalities for sums of bounded random variables. J. Amer. Statist. Assoc. 58, 13-30.

[22] Kane, C., Marsden, J. E., Ortiz, M., 1999. Symplectic-energymomentum preserving variational integrators. Journal of Mathematical Physics 40 (7), 3353-3371.

[23] Kidane, A., Lashgari, A., Li, B., McKerns, M., Ortiz, M., Owhadi, H., Ravichandran, G., Stalzer, M., Sullivan, T. J., 2012. Rigorous modelbased uncertainty quantification with application to terminal ballistics. Part I: Systems with controllable inputs and small scatter. Journal of the Mechanics and Physics of Solids 60 (5), 983-1001. 
[24] Leonard, T., Hsu, J. S. J., 1999. Bayesian methods. Vol. 5 of Cambridge Series in Statistical and Probabilistic Mathematics. Cambridge University Press, Cambridge, an analysis for statisticians and interdisciplinary researchers.

[25] Li, B., Habbal, F., Ortiz, M., 2010. Optimal transportation meshfree approximation schemes for fluid and plastic flows. International Journal for Numerical Methods in Engineering 83 (12), 1541-1579.

[26] Li, B., Kidane, A., Ravichandran, G., Ortiz, M., 2012. Verification and validation of the Optimal Transportation Meshfree (OTM) simulation of terminal ballistics. International Journal of Impact Engineering 42, $25-36$.

[27] Li, B., Mihaly, J., Rosakis, A., Ortiz, M., 2014. Modeling, simulation and validation of hypervelocity impact, submitted for publication.

[28] Liu, J. S., 2008. Monte Carlo strategies in scientific computing. Springer Series in Statistics. Springer, New York.

[29] Lucas, L. J., Owhadi, H., Ortiz, M., 2008. Rigorous verification, validation, uncertainty quantification and certification through concentrationof-measure inequalities. Comput. Methods Appl. Mech. Engrg. 197 (5152), 4591-4609.

[30] Marsh, S. P. (Ed.), 1980. LASL Shock Hugoniot Data. Los Alamos Series on Dynamic Material Properties. University of California Press.

[31] McDiarmid, C., 1989. On the method of bounded differences. In: Surveys in combinatorics, 1989 (Norwich, 1989). Vol. 141 of London Math. Soc. Lecture Note Ser. Cambridge Univ. Press, Cambridge, pp. 148188.

[32] McKerns, M., Hung, P., Aivazis, M., 2009. Mystic: A simple model-independent inversion framework. http://dev.danse.us/trac/mystic.

[33] McKerns, M., Owhadi, H., Scovel, C., Sullivan, T. J., Ortiz, M., 2012. The optimal uncertainty algorithm in the mystic framework. arXiv 1202.1055, http://arxiv.org/abs/1202.1055.

[34] Mihaly, J. M., 2013. Investigation of hypervelocity impact phenomena using real-time concurrent diagnostics. Ph.D. thesis, California Institute of Technology. 
[35] Mihaly, J. M., Tandy, J. D., Adams, M. A., Rosakis, A. J., 2013. In situ diagnostics for a small-bore hypervelocity impact facility. International Journal of Impact Engineering 62, 13-26.

[36] Niederreiter, H., 1992. Random number generation and quasi-Monte Carlo methods. Vol. 63 of CBMS-NSF Regional Conference Series in Applied Mathematics. Society for Industrial and Applied Mathematics (SIAM), Philadelphia, PA.

[37] Owhadi, H., Scovel, C., Sullivan, T. J., McKerns, M., Ortiz, M., 2013. Optimal Uncertainty Quantification. SIAM Review 55 (2), 271-345.

[38] Pandolfi, A., Ortiz, M., 2012. An eigenerosion approach to brittle fracture. International Journal for Numerical Methods in Engineering $92(8), 694-714$.

[39] Price, K., Storn, R., 1997. Differential evolution. Dr Dobbs Journal $22(4), 18^{-+}$.

[40] Price, K. V., Storn, R. M., Lampinen, J. A., 2005. Differential Evolution: A Practical Approach to Global Optimization. Natural Computing Series. Springer-Verlag, Berlin.

[41] Saltelli, A., Chan, K., Scott, E. M. (Eds.), 2000. Sensitivity Analysis. Wiley Series in Probability and Statistics. John Wiley \& Sons Ltd., Chichester.

[42] Saltelli, A., Ratto, M., Andres, T., Campolongo, F., Cariboni, J., Gatelli, D., Saisana, M., Tarantola, S., 2008. Global Sensitivity Snalysis. The Primer. John Wiley \& Sons Ltd., Chichester.

[43] Schmidt, B., Fraternali, F., Ortiz, M., 2009. Eigenfracture: An eigendeformation approach to variational fracture. Multiscale Modeling \& Simulation 7 (3), 1237-1266.

[44] Shen, X., Wasserman, L., 2001. Rates of convergence of posterior distributions. Ann. Statist. 29 (3), 687-714.

URL http://dx.doi.org/10.1214/aos/1009210686

[45] Sloan, I. H., 2010. Sparse sampling techniques. Presented at 2010 ICMS Uncertainty Quantification workshop.

URL http://icms.org.uk/downloads/uq/Sloan.pdf 
[46] Sloan, I. H., Joe, S., 1994. Lattice methods for multiple integration. Oxford Science Publications. The Clarendon Press Oxford University Press, New York.

[47] Soekkha, H. M. (Ed.), 1997. Aviation Safety: Human Factors, System Engineering, Flight Operations, Economics, Strageties, Management. VSP.

[48] Stainier, L., Ortiz, M., 2010. Study and validation of a variational theory of thermo-mechanical coupling in finite visco-plasticity. International Journal of Solids and Structures 47 (5), 705-715.

[49] Steinberg, D. J., February 1996. Equation of State and Strength Properties of Selected Materials. Tech. rep., Lawrence Livermore National Laboratory.

[50] Storn, R., Price, K., 1997. Differential evolution-a simple and efficient heuristic for global optimization over continuous spaces. J. Global Optim. 11 (4), 341-359, http://dx.doi.org/10.1023/A:1008202821328.

[51] Stuart, A. M., 2010. Inverse problems: a Bayesian perspective. Acta Numer. 19, 451-559.

URL http://dx.doi.org/10.1017/S0962492910000061

[52] Sullivan, T. J., McKerns, M., Meyer, D., Theil, F., Owhadi, H., Ortiz, M., 2013. Optimal uncertainty quantification for legacy data observations of Lipschitz functions. ESAIM Math. Model. Numer. Anal. 47 (6), 1657-1689.

URL http://dx.doi.org/10.1051/m2an/2013083

[53] Sullivan, T. J., Topcu, U., McKerns, M., Owhadi, H., 2011. Uncertainty quantification via codimension-one partitioning. Int. J. Num. Meth. Engrg. 85 (12), 1499-1521.

[54] Sulsky, D., Chen, Z., Schreyer, H. L., 1994. A particle method for history-dependent materials. Computer Methods in Applied Mechanics and Engineering 118 (1-2), 179-196.

[55] Todor, R. A., Schwab, C., 2007. Convergence rates for sparse chaos approximations of elliptic problems with stochastic coefficients. IMA J. Numer. Anal. 27 (2), 232-261.

URL http://dx.doi.org/10.1093/imanum/dr1025 
[56] Van Duin, A., 2002. ReaxFF User Manual. California Institute of Technology, Materials and Process Simulation Center.

[57] Villani, C., 2009. Optimal Transport: Old and New. Vol. 338 of Grundlehren der Mathematischen Wissenschaften [Fundamental Principles of Mathematical Sciences]. Springer-Verlag, Berlin.

URL http://dx.doi .org/10.1007/978-3-540-71050-9

[58] Xiu, D., 2009. Fast numerical methods for stochastic computations: a review. Commun. Comput. Phys. 5 (2-4), 242-272.

[59] Yadav, S., Repetto, E. A., Ravichandran, G., Ortiz, M., 2001. A computational study of the influence of thermal softening on ballistic penetration in metals. International Journal of Impact Engineering 25 (8), 787-803.

[60] Yang, Q., Stainier, L., Ortiz, M., 2006. A variational formulation of the coupled thermo-mechanical boundary-value problem for general dissipative solids. Journal of the Mechanics and Physics of Solids 54 (2), 401-424. 